\title{
An Integrated Analysis of Network Pharmacology and Experimental Validation to Reveal the Mechanism of Chinese Medicine Formula Naotaifang in Treating Cerebral Ischemia-Reperfusion Injury
}

\author{
Tong Yang' \\ Xiangyu Chen' \\ Zhigang Mei (iD ${ }^{1,2}$ \\ Xiaolu Liu ${ }^{2}$ \\ Zhitao Feng $\mathbb{( D}^{2}$ \\ Jun Liao' \\ Yihui Deng' \\ Jinwen $\mathrm{Ge} \mathbb{D}^{\prime}$
}

'Key Laboratory of Hunan Province for Integrated Traditional Chinese and Western Medicine on Prevention and Treatment of Cardio-Cerebral Diseases, College of Integrated Traditional Chinese and Western Medicine, Hunan University of Chinese Medicine, Changsha, Hunan, People's Republic of China; ${ }^{2}$ Third-Grade Pharmacological Laboratory on Chinese Medicine Approved by State Administration of Traditional Chinese Medicine, Medical College of China Three Gorges University, Yichang, Hubei, People's Republic of China
Correspondence: Zhigang Mei; Jinwen Ge College of Integrated Traditional Chinese and Western Medicine, Hunan University of Chinese Medicine, NO. 300, Xueshi Road, Yuelu District, Changsha, Hunan, 410218, People's Republic of China Email meizhigang@hnucm.edu.cn; 00I267@hnucm.edu.cn
Background: Cerebral ischemia-reperfusion injury (CIRI) is a crucial factor leading to a poor prognosis for ischemic stroke patients. As a novel Chinese medicine formula, Naotaifang (NTF) was proven to exhibit a neuroprotective effect against ischemic stroke, clinically, and to alleviate CIRI in animals. However, the mechanisms underlying the beneficial effect have not been fully elucidated.

Methods: In this study, we combined a network pharmacology approach and an in vivo experiment to explore the specific effects and underlying mechanisms of NTF in the treatment of ischemia-reperfusion injury. A research strategy based on network pharmacology, combining target prediction, network construction, gene ontology (GO), Kyoto Encyclopedia of Genes and Genomes (KEGG) pathway enrichment analysis, and molecular docking was used to predict the targets of NTF in treating the ischemic stroke and CIRI. On the other hand, we used HPLC and HRMS to identify biologically active components of NTF. Middle cerebral artery occlusion models in rats were utilized to evaluate the effect and the underlying mechanisms of NTF against CIRI after ischemic stroke.

Results: Network pharmacology analysis revealed 43 potential targets and 14 signaling pathways for the treatment of NTF against CIRI after ischemic stroke. Functional enrichment analysis showed that a STAT3/PI3K/AKT signaling pathway serves as the target for in vivo experimental study validation. The results of animal experiments showed that NTF significantly alleviated CIRI by decreasing neurological score, infarct volume, numbers of apoptotic neuronal cells, increasing density of dendritic spines and survival of neurons. Furthermore, NTF could increase the expression of p-STAT3, PI3K, p-AKT. In addition, the detection of apoptosis-related factors showed that the NTF could raise the expression of Bcl-2 and reduce the expression of Bax.

Conclusion: This network pharmacological and experimental study indicated that NTF, as a therapeutic candidate for the management of CIRI following ischemic stroke, may exert a protective effect through the STAT3/PI3K/AKT signaling pathway.

Keywords: cerebral ischemia-reperfusion injury, stroke, network pharmacology, molecular docking, STAT3/PI3K/AKT signaling pathway

\section{Introduction}

Stroke is a serious threat to human health and the second leading cause of death worldwide owing to its high morbidity, mortality, and disability rates. Currently, stroke is a major cause of death in China, ${ }^{1}$ and approximately $87 \%$ of strokes are ischemic 
strokes, which are characterized by a sudden loss of blood circulation in a certain area of the brain, leading to the corresponding loss of neurologic function. ${ }^{2}$ Timely restoration of blood flow is one of the important treatment principles in clinical application. ${ }^{3}$ At present, recombinant tissue plasminogen activator (rtPA) remains the only recommended pharmacotherapy for acute ischemic stroke approved by the American Food and Drug Administration (FDA). However, due to the limitation of a narrow therapeutic time window and high risk of hemorrhagic transformation, only $2-10 \%$ globally and $1.2-3.8 \%$ in China, ischemic stroke patients benefit from the thrombolytic therapy. ${ }^{4-6}$ Furthermore, blood flow recanalization could lead to cerebral ischemia-reperfusion injury (CIRI). CIRI involves multiple mechanisms, such as inflammation, oxidative stress, calcium overload, cell apoptosis, and glutamate excitotoxicity, which are defined as biochemical cascade reactions leading to further deterioration of ischemic brain tissue, thereby reversing the benefits of restoring circulation following stroke occurrence. ${ }^{7,8}$ Therefore, it is crucial to explore an alternative or complementary medicine including traditional ethnical medicine to prevent or alleviate CIRI in ischemic stroke patients.

Traditional Chinese medicine (TCM) is characterized by its systematic and holistic concept. The systematic nature of this strategy is consistent with the holistic view of Chinese medicine and the multi-component, multi-target, and multipathway synergistic mechanism of Chinese medicine formulas. $^{9-11}$ From TCM monomers $^{12}$ to TCM extract preparations, ${ }^{13}$ as the alternative or complementary medicine, TCM has been used in China and other countries to treat ischemic stroke for hundreds of years. TCM has achieved a lot of advantages due to their multiple pharmaceutical targets and fewer side effects. ${ }^{14-16}$ Naotaifang (NTF), a novel traditional Chinese formula, which is simplified and adapted from a well-known TCM prescription Buyang Huanwu decoction (BYHWD). The recipe for NTF consists of four natural medicines (Table 1), ie Radix Astragali (Huangqi, HQ), Rhizoma Ligustici Chuanxiong (Chuanxiong, CX), Pheretima (Dilong, DL), Bombyx Batryticatus (Jiangcan, JC). ${ }^{17-19}$ Clinical evidence showed that NTF exerts anti-ischemic stroke effects, such as mitigating the neurological symptoms of patients with acute cerebral ischemia. ${ }^{19,20}$ Previous animal experiments have well demonstrated that NTF can meliorate CIRI through various mechanisms. ${ }^{21-23}$ For instance, the formula has been shown to improve blood circulation, anticoagulation, angiogenesis, anti-peroxide, anti-inflammation and
Table I Pharmaceutical Ingredients of NTF

\begin{tabular}{|l|l|l|l|}
\hline $\begin{array}{l}\text { Latin } \\
\text { Name }\end{array}$ & Species & Family & Part Used \\
\hline $\begin{array}{l}\text { Radix } \\
\text { Astragali }\end{array}$ & $\begin{array}{l}\text { Astragalus } \\
\text { membranaceus } \\
\text { (Fisch.) Bge.var. } \\
\text { Mongholicus (Bge.) } \\
\text { Hsiao }\end{array}$ & Leguminosae & Roots \\
\hline $\begin{array}{l}\text { Rhizoma } \\
\text { Ligustici }\end{array}$ & $\begin{array}{l}\text { Ligusticum chuanxiong } \\
\text { Hort. }\end{array}$ & Umbelliferae & Rhizomes \\
\hline $\begin{array}{l}\text { Pheretima } \\
\text { Pheretima aspergillum } \\
\text { (E. Perrier) }\end{array}$ & Megascolecidae & Bodies \\
\hline Batryticatus & $\begin{array}{l}\text { Bombyx mori } \\
\text { Linnaeus. }\end{array}$ & Bombycidae & $\begin{array}{l}\text { Bodies infected } \\
\text { by Beauveria } \\
\text { bassiana (Bals.) } \\
\text { Vuillamt }\end{array}$ \\
\hline
\end{tabular}

protect neurons from programmed cell deaths including apoptosis and ferroptosis. ${ }^{16,19-23}$ However, the precise mechanisms underlying the effect of NTF have only been partially unraveled, and further comprehensive and systematic research is needed before it can be widely applied in the treatment of CIRI.

Network pharmacology, which is a method to research the potential mechanisms of the formula in living organisms, elucidating the interaction between formula and disease with the aim of providing supporting evidence for experimental studies. $^{24}$ The approach's systemic and holistic traits are in line with the concept of TCM, through which, this study could have a systematic and integrative viewpoint on the intervention and impact of NTF on ischemic stroke and CIRI network. Furthermore, this study established the comprehensive network pharmacology approach and molecular docking strategy, combined with in vivo experiments to explore the potential key targets and possible pharmacological mechanisms of the effect of NTF against CIRI, providing a scientific basis for the predicted mechanisms.

\section{Materials and Methods}

Prediction of the Mechanism of NTF Intervention in Ischemic Stroke by Network Pharmacology and Molecular Docking Compound Database Construction for NTF

Chemical compounds in NTF were retrieved from Traditional Chinese Medicine Systems Pharmacology Database and Analysis Platform (TCMSP), and The 
TCM Database@ @Taiwan (http://tcm.cmu.edu.tw/zh-tw/). Then, the compounds that may be substandard but important were screened out through large-scale text mining. The active compounds of NTF were mainly filtered with OB and DL. Next, we filtered out compounds that may not match the criteria but were important components by text mining. The framework of this research is shown in Figure 1.

\section{Target Prediction}

To provide the scientific and coherent biological basis between the targets of NTF, ischemic stroke and CIRI, the target proteins of the active compounds in NTF were from TCMSP, PubChem (https://pubchem.ncbi.nlm.nih. $\underline{\text { gov/) }}$ and Swiss Target Prediction (http://www.swisstarget prediction.ch/) databases with the species limited to "Homo sapiens". The standard gene names of targets which were related with compounds were obtained from the UniProt database (https://www.uniprot.org/). And we acquired the genes related with ischemic stroke from the Genecards (https://www.genecards.org/) and the Online Mendelian Inheritance in Man (OMIM) (https://www. omim.org/) databases. Thus, we inquired the target genes associated with drugs and disease, which means that they could be better to elucidate the role of NTF against ischemic stroke and CIRI through the intersection between target genes of active compounds in NTF and relevant genes related with ischemic stroke including CIRI.

\section{PPI Data and Network Construction}

To provide the scientific and reasonable interpretation of the complex relationships between the targets of NTF, ischemic stroke and CIRI, we matched the prediction of

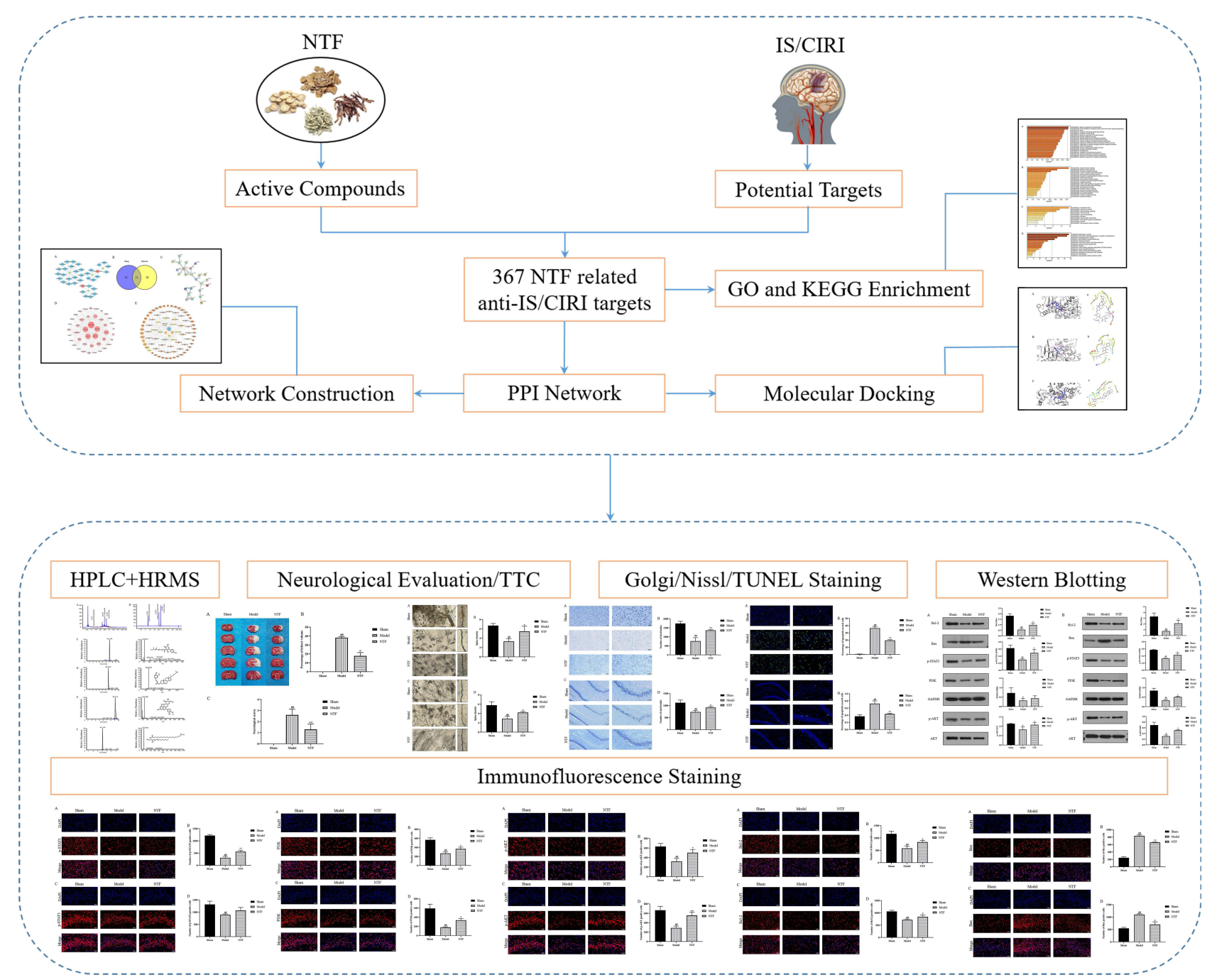

Figure I The flowchart of network pharmacology and molecular docking-based strategy for deciphering the underlying mechanisms of NTF on the treatment of IS/CIRI. 
the targets of active compounds in NTF and the retrieval of the related targets of ischemic stroke and CIRI, then chose the overlapping targets as the related targets of NTF in treating ischemic stroke and CIRI. The targets were then acquired by String (https://string-db.org/) with the species limited to "Homo sapiens" and a confidence score $>0.99$ to draw the data of PPI, which was a database to predict protein interactions, including direct and indirect interactions, and to evaluate the interaction information. ${ }^{25}$ All visualized network models were established via Cytoscape 3.7.2, which was an open software package project to visualize, integrate, model and analyze the interaction networks. ${ }^{26}$ The proteins derived from proteincoding genes locus were represented by nodes in the interaction network.

We selected three parameters to assess its topological features: (1) Degree centrality (DC) was defined as the number of connections between one node and other nodes. (2) Betweenness centrality (BC) was the ratio of the number of paths through the nodes of all shortest paths in the network to the total number of shortest paths, which measures the extent to which a node lies on paths between other nodes. (3) Closeness centrality (CC) reflected the mean distance from a node to other nodes. The three parameters played a crucial role in the network, as well as the important indicators for new drug discovery and target prediction. ${ }^{27}$ The corresponding median values of each parameter was regarded as the threshold values to analyze hub nodes in the network. ${ }^{28}$ According to these data, we constructed three networks in this study: (1) herbcompound network; (2) key targets network; (3) key target-compound network. However, the limitation of this study is that the interaction relationships of the nodes in these networks remains unclear. For example, the type of action such as activation, inhibition, binding, catalysis, the effect of action such as positive, negative, unspecified, and so on.

\section{Molecular Docking}

All structures of target proteins were downloaded from the RCSB PDB database (http://www.pdb.org), and all those for ingredients of NTF were obtained from the PubChem database (https://pubchem.ncbi.nlm.nih.gov/). Molecular docking was performed by using the Protein Preparation, Ligprep, SiteMap, Glide modules of the Schrodinger. Target proteins as reporters were prepared by the Protein Preparation module in the Schrodinger, including the removal of water molecules and energy minimization.
Similarly, target genes as ligands were prepared by using the Ligprep module of Schrodinger. ${ }^{29}$ The SiteMap module of Schrodinger predicted the possible active binding site of receptors. ${ }^{30,31}$ The Glide module was used for analyzing the ligand-reporter binding affinity and interactions $^{29}$ and the proteins with the absolute value of Glide score greater than 3 were chosen as the proteins with good effect. ${ }^{32}$ The docking score of each ligand was determined from the best pose by visual evaluation on Maestro(Schrodinger). ${ }^{33}$

\section{GO and KEGG Pathway Enrichment Analysis}

Metascape was a web-based portal designed to provide experimental biologists with a comprehensive gene list annotation and analysis resource. ${ }^{34}$ To systematically explore the concerned biological processes of NTF as a therapy against ischemic stroke, we used Metascape (http://metascape.org/ gp/index.html\#/main/step1) as a stateful web service to facilitate gene list analysis for GO enrichment analysis and KEGG pathway enrichment analysis. According to Fisher's exact test, GO and KEGG pathway with $P$ value less than 0.05 were considered statistically significant, and further analysis was conducted.

\section{Medicine Preparation NTF Extract}

NTF consists of four herbs: HQ, CX, DL, JC with ratio $8: 2: 3: 3$. In our present study, drugs were purchased from the First Hospital of Hunan University of Chinese Medicine, Hunan province, China. A voucher specimen was deposited (dry conditions, normal temperature) in the College of Integrated Traditional Chinese and Western Medicine of Hunan University of Chinese Medicine, Hunan province, China. The dried plant material was extracted two times with ultrapure water $(1: 10)$ in flask heating mantles at $100{ }^{\circ} \mathrm{C}, 1.5-2$ hours each time and then filtered through a two-layer filter screen and then concentrated to $10 \%$ by rotary evaporator and collected the final solution $(10 \%)$. NTF extract was stored at $4{ }^{\circ} \mathrm{C}$ before experiment. According to previous researches and clinical administration dose, the rats in the NTF group were administered with NTF extract (NTF, $9 \mathrm{~g} / \mathrm{kg}$ per day). ${ }^{16,23}$

\section{High-Performance Liquid Chromatography}

In order to verify the quality of NTF, we used high performance liquid chromatography (HPLC) to qualitatively verify three representative compounds in NTF: ligustrazine hydrochloride, calycosin 7-o-glucoside, and ferulic acid. 
The chromatographic column consisted of the following: Welch Ultimate XB C18 $(4.6 \times 250 \mathrm{~mm}, 5 \mu \mathrm{m})$, moving phase A: water with $0.1 \%$ trifluoroacetic acid, moving phase B: $0.1 \%$ trifluoroacetic acid in acetonitrile, gradient elution flow rate: $1 \mathrm{~mL} / \mathrm{min}$, detection wavelengths: DAD $249 \mathrm{~nm}$, and injection volume: $2 \mu \mathrm{L}$. The gradient elution was 0-5.0 min, $95 \% \mathrm{~A}: 10 \% \mathrm{~B}$; $5.0-15.0 \mathrm{~min}, 0 \% \mathrm{~A}: 70 \% \mathrm{~B}$; 15.0-15.1 min, 0\%A: 90\%B; 15.1-20.0 min, 0\%A: 90\%B; 20.0-20.1 min, 95\%A: 10\%B; 20.1-25.0 min, 95\%A: 10\% B. Data were acquired and analyzed using ChemStatio software.

\section{High Resolution Mass Spectrometer}

A sensitive and rapid high-resolution mass spectrometer (HRMS) was also used to analyze the active ingredients that the NTF contained. The chromatographic separation was achieved by using RP C18 column $(150 \times 2.1 \mathrm{~mm}$, $1.8 \mu \mathrm{m}$ ), which was maintained at $35{ }^{\circ} \mathrm{C}$. The mobile phase consisted of $0.1 \%$ formic acid (A) and acetonitrile (B). The gradient elution flow rate: $0.3 \mathrm{~mL} / \mathrm{min}$, and injection volume: $5 \mu \mathrm{L}$. The gradient elution was $0-0.5$ min, $98.0 \% \mathrm{~A}: 2.0 \% \mathrm{~B}$; $0.5-6.5 \mathrm{~min}, 2.0 \% \mathrm{~A}: 98.0 \% \mathrm{~B}$; 6.5-9.0 $\mathrm{min}, 2.0 \% \mathrm{~A}: 98.0 \% \mathrm{~B} ; 9.0-9.3 \mathrm{~min}, 98.0 \% \mathrm{~A}$ : $2.0 \% \mathrm{~B} ; 9.3-10.0 \mathrm{~min}, 98 \% \mathrm{~A}: 2 \% \mathrm{~B}$. The optimized mass parameters were set as follows: electrospray ionization (ESI); scan mode: positive and negative; scan range, $50.0-500.0 \mathrm{~m} / \mathrm{z}$; spray voltage, $3.2 \mathrm{Kv}$ (positive); capillary temperature, $300{ }^{\circ} \mathrm{C}$; collision gas, argon gas ( $\geq 99.999 \%)$; sheath gas, nitrogen $(\geq 99.999 \%)$; auxiliary gas, nitrogen $(\geq 99.999 \%), 350{ }^{\circ} \mathrm{C}$. Constituents were identified by HRMS in negative ion mode using full scan and two-stage threshold-triggered mass modes.

\section{Experimental Methods}

\section{Animal Grouping and Treatment}

The fifty-four adult male Sprague-Dawley rats (weighing 220-250 g, 6-8 weeks old, 110727201100883051, 11072721100835278 and 1107272011007367$)$ were purchased by the Animal Center of Hunan University of Chinese Medicine (Changsha, China) with approval number SYXK 2019-0009. All procedures regarding animals in this study were in compliance with and approved by the Ethics Committee of Hunan University of Chinese Medicine, and in accord with the guidelines of the National Institutes of Health on the care and use of animals. All rats were randomly assigned into three groups (n $=18$ for each group), including the sham group, model group, NTF group. Total rats had acclimatisation periods of 5 days before the formal experiments. The rats in the NTF group were given NTF extract by gavage and rats in the other groups were given an equal volume of physiological saline by gavage once daily for 7 days before transient middle cerebral artery occlusion. The rats were housed in a room temperature environment and resumed normal diet after surgery. CIRI were induced by transient middle cerebral artery occlusion surgery in all rats except for the sham group. The intraluminal filament was used to block the middle cerebral artery, and the blood flow was restored after $1.5 \mathrm{~h}$. According to the requirements of the Experimental Animal Management Committee of Hunan University of Chinese Medicine, if an animal becomes seriously ill during experiment process, it must be humanely killed by inhalation of $\mathrm{CO}_{2}$ in order to prevent the animal from suffering.

\section{The Procedures of CIRI}

After the pre-administration with NTF extract for 7 days, an MCAO model was established to simulate CIRI successfully in accordance with the method in our previous researches. ${ }^{7,35}$ Briefly, the rats were anesthetized by the intraperitoneal (ip) injection of $10 \%$ chloral hydrate, at the dose of $3.5 \mathrm{~mL} / \mathrm{kg}$. After fixation and disinfection, all the right common carotid artery, external carotid artery and internal carotid artery were exposed, and the proximal end of the common carotid artery was ligated. A nylon monofilament, about $40 \mathrm{~mm}$ long and 0.24 $0.26 \mathrm{~mm}$ in diameter, was inserted from the right common carotid artery to the lumen of internal carotid artery with an insertion depth of $22 \pm 0.5 \mathrm{~mm}$ to block the origin of the right middle cerebral artery according to the weight and the carotid diameter of the animal subjected to surgery. After 90 min of occlusion, the inserted filament was carefully removed to restore blood flow. After modeling, the rats were put back in the same environment.

\section{Neurological Evaluation}

Five levels of neurological evaluation were evaluated according to Longa et $\mathrm{al}^{36}$ scale in a randomly selected 10 rats per group of observers who were unaware of the animal grouping system $24 \mathrm{~h}$ after reperfusion on the following scale. Score 0: normal, no neurological deficit; score 1: inability to fully extend the left front paw, mild neurological deficit; score 2: the rat turned to the left side (temporal side) while walking, moderate neurological deficit; score 3: the rat inverted to the left side (temporal side) 
while walking, severe neurological deficit and score 4: inability to walk, loss of consciousness, score more than 3 points or elimination of the rat.

\section{TTC Staining}

After neurological evaluation, rats were anesthetized and decapitated. Brain tissues were quickly removed from the skull and then cut into five 2-mm-thick coronal sections, which were frozen at $-20{ }^{\circ} \mathrm{C}$ for 50 minutes before all tissue sections were stained with $2 \%$ TTC at $37^{\circ} \mathrm{C}$ for 30 minutes and then fixed overnight in $4 \%$ paraformaldehyde. The infarct area was shown in white and the non-infarcted portion in red. Infarct volume was analyzed using Imagepro plus 6.0 which was unknown to the experimental group. To exclude the effect of cerebral edema, the infarct area was normalized to the non-ischemic hemisphere and expressed as a percentage of the contralateral hemisphere.

\section{Golgi Staining}

The Golgi-Cox staining procedure used here was based on previous studies. ${ }^{37}$ The brains were stored in the dark for 2 days and then transferred into fresh Golgi-Cox solution for 14 days. Dehydration in continuous alcohol baths of $50 \%$ ( $2 x / 5 \mathrm{~min}), 70 \%(2 \mathrm{x} / 5 \mathrm{~min}), 90 \%(3 \mathrm{x} / 5 \mathrm{~min})$, and $100 \%$ $(3 \mathrm{x} / 5 \mathrm{~min})$, followed by xylene baths $(2 \mathrm{x} / 10 \mathrm{~min})$. All slides were then fixed, covered with coverslips and kept in a dark environment for 15 days without any manipulation. The dendritic spines were observed in and the dendritic spine density of pyramidal neurons were analyzed in the ischemic area.

\section{Nissl Staining}

Right hemisphere tissue sections were taken for Nissl staining after $24 \mathrm{~h}$ of reperfusion. The above-prepared sections were dewaxed, rehydrated, immersed in toluidine blue (Servicebio, China) solution for $5 \mathrm{~min}$, rinsed with distilled water, dehydrated with ethanol and xylene, and then cover slipped with neutral balsam. The cytoplasm of the stained cells in the cortex and hippocampus of the rat brains were observed to turn purple-blue and the nuclei were light blue under optical microscope. The number of Nissl bodies in the cortical area was quantified.

\section{TUNEL Staining}

According to the manufacturer's instructions, a terminal deoxynucleotidyl transferase-mediated dUTP-biotin nick end labeling (TUNEL) assay was used to determine the apoptosis-positive cells in rat brain. Paraffin-embedded sections of rat brain tissue were blocked with $3 \%$ hydrogen peroxide for $10 \mathrm{~min}$ and incubated at $37{ }^{\circ} \mathrm{C}$ in buffer for $20 \mathrm{~min}$. The sections were added with terminal deoxynucleotidyl transferase reaction mixture at $37{ }^{\circ} \mathrm{C}$ for $2 \mathrm{~h}$, incubated with a POD peroxidase labeling reagent at $37{ }^{\circ} \mathrm{C}$ for $30 \mathrm{~min}$ and then stained with 4'6-diamidino-2-phenylindole (DAPI). Sections were not incubated with terminal deoxynucleotidyl transferase reaction mix in negative control tissue. TUNEL-positive cells were in green with nucleus DAPI staining in blue. The percentage of TUNEL positive (apoptotic) cells apoptotic index of nonoverlapping brain tissue was calculated.

\section{Immunofluorescence Staining}

All brain tissues were embedded in paraffin. Each paraffin section was fixed with $4 \%$ paraformaldehyde for 12 min, $0.25 \%$ Triton for $5 \mathrm{~min}$, blocked with $1 \%$ BSA diluted in PBS for 30 min at room temperature, and then with primary antibody anti-Bcl-2, anti-Bax, anti-p-STAT3, anti-PI3K, anti-p-AKT, anti-AKT (dilution of 1:1000) and NeuN overnight at $4{ }^{\circ} \mathrm{C}$. After rinsing 3 times with PBST, sections were incubated with Cy3-conjugated anti-rabbit IgG (dilution of 1:300, Servicebio Co., Ltd., China) for 50 min at room temperature, followed by incubation with DAPI (dilution of 1:300, Servicebio Co., Ltd., China) for $10 \mathrm{~min}$. Total positive cells were stained in red with nucleus DAPI staining in blue. All sections were observed by a researcher who did not understand the experiment design with a fluorescence microscope (Nikon, Japan), including cover lipping, imaging and photographing. Each experimental group included at least three brain sections for staining examinations.

\section{Western Blotting}

The ischemic penumbra of the cerebral cortex and hippocampus were obtained for Western blotting analysis, which were harvested at $24 \mathrm{~h}$ reperfusion and stored in a $-80{ }^{\circ} \mathrm{C}$ refrigerator, and the tissues were lysed with RIPA buffer. The tissue samples were centrifuged at $12,000 \mathrm{~g}$ for 15 min, and the supernatants were collected and boiled. The protein concentrations were determined with a spectrophotometer, and then subjected to sodium dodecyl sulfate-polyacrylamide gel electrophoresis. The nitrocellulose membrane was blocked with skimmed milk (5\%) in Tris-buffered saline containing Tween and then it was incubated with antibodies against Bcl-2, Bax, p-STAT3, PI3K, p-AKT and AKT overnight at $4{ }^{\circ} \mathrm{C}$. Next, the tissue was incubated with secondary antibodies for $120 \mathrm{~min}$ at 
room temperature, rewashed with TBST, and the protein bands were detected using the CLINX 6300 imaging system.

\section{Statistical Analysis}

All data were analyzed using SPSS 25.0 statistical software. Quantitative data were expressed as the mean \pm standard deviation (SD). The significant differences between the groups were examined by one-way analysis of variance (ANOVA) with the least significant difference test. Values of $p<0.05$ were considered to be statistically significant.

\section{Results}

\section{Analysis of NTF Ingredients}

Three representative compounds and four active ingredients in NTF had been verified respectively by HPLC and HRMS, which are shown in Figure 2. The main compounds were quantified: ligustrazine hydrochloride 2.07 $\mu \mathrm{g} / \mathrm{mL}$, calycosin 7-o-glucoside $176.23 \mu \mathrm{g} / \mathrm{mL}$, and ferulic acid $286.96 \mu \mathrm{g} / \mathrm{mL}$. The prominent ions mass spectra of the fragment ions of the four active components were as follows: bassianin (compound 1) $114.09 \mathrm{~m} / \mathrm{z}$, cholesteryl ferulate (compound 2) $269.08 \mathrm{~m} / \mathrm{z}$, hyrcanoside (compound 3) $133.10 \mathrm{~m} / \mathrm{z}$ and (4E,6E,2S,3R)-2-N-docosanoyl4,6-tetradecasphingadienine (compound 4) $137.05 \mathrm{~m} / \mathrm{z}$. The above analysis and the standard chemical structures of compounds 1, 2, 3 and 4 showed that the NTF extracts contained bassianin, cholesteryl ferulate, hyrcanoside and (4E,6E,2S,3R)-2-N-docosanoyl-

4,6-tetradecasphingadienine.

\section{Prediction of Network Pharmacology of NTF on Ischemic Stroke and CIRI}

Predictions of NTF on ischemic stroke and CIRI were investigated by network analysis as shown in Figure 3. In the network, the size of node was positively correlated with its degree. In order to clarify the relationship between the herbs and potential active compounds, the herb-compound network of NTF is constructed in Figure 3A, from which we could find out that the FA (MOL000433), cholesteryl ferulate, etc. were predicted as the important active compounds in NTF. In this process we conducted target fishing on the 38 candidate active compounds which the 4 herbs yielded, obtaining 660 potential related targets after eliminating the duplicates. Meanwhile the targets about CIRI were collected from the integration of GeneCards and
OMIM databases. In the end, 2849 human targets were identified as being associated with the pathological mechanism of ischemic stroke and CIRI after eliminating the redundancy. Further analysis revealed that 367 targets were shared between 660 combined targets and 2849 disease targets in Figure 3B. In the database of String, a PPI network was established, among which 367 matching targets were regarded as the related targets of NTF and CIRI after two screenings, whose interaction scores were the highest confidence scores $(>0.99)$. There were 367 nodes and 2293 edges in total. The topological feature analysis of the PPI network was based on three major parameters of $\mathrm{DC}, \mathrm{BC}$ and $\mathrm{CC}$ which were calculated by the CytoNCA plug-in for Cytoscape 3.7.2. According to these values, we selected the median of them as potential drug targets for subsequent analysis, and the results settled at $116 \mathrm{key}$ targets as $\mathrm{DC} \geq 16, \mathrm{BC} \geq 0.004$ and $\mathrm{CC} \geq 0.48$, and the results settled at 43 hub nodes and 310 edges, to better understand the picture more clearly, we showed the picture as $p>0.99$ (Figure 3C), which were listed in Table 2. And the graphical interactions of the 43 key targets were visualized in Figure 3D, among which PIK3CA (degree $=29$ ), STAT3, $($ degree $=25)$, SRC $($ degree $=25)$, MAPK1 (degree $=25)$, MAPK3 $($ degree $=25)$ were key targets in this network. According to the related potential active components, we constructed the herb-compound-target network based on 43 key targets (Figure 3E). In this network, the top seven compounds were ecdysterone, which holds relevancy to 14 key targets; bassianin, which held relevancy to 13 key targets; cholesteryl ferulate, which was related to 12 key targets; beauvericin, related to 11 key targets; hyrcanoside, ergotamine and lupeol acetate were associated with 10 key targets.

\section{Characterization of Potential Therapeutic Targets of NTF}

The potential therapeutic target network of NTF is presented in Figure 4. GO enrichment analysis of 43 potential key targets for NTF treating ischemic stroke and CIRI were performed, and the biological process (Figure 4A), molecular function (Figure 4B) and cellular component (Figure 4C) were included. We found that the top ten biological processes terms $(p<0.05)$ were related to the positive regulation of locomotion, transmembrane receptor protein tyrosine kinase signaling pathway, taxis, cytokine-mediated signaling pathway, response to wounding, positive regulation of kinase activity, cellular response 

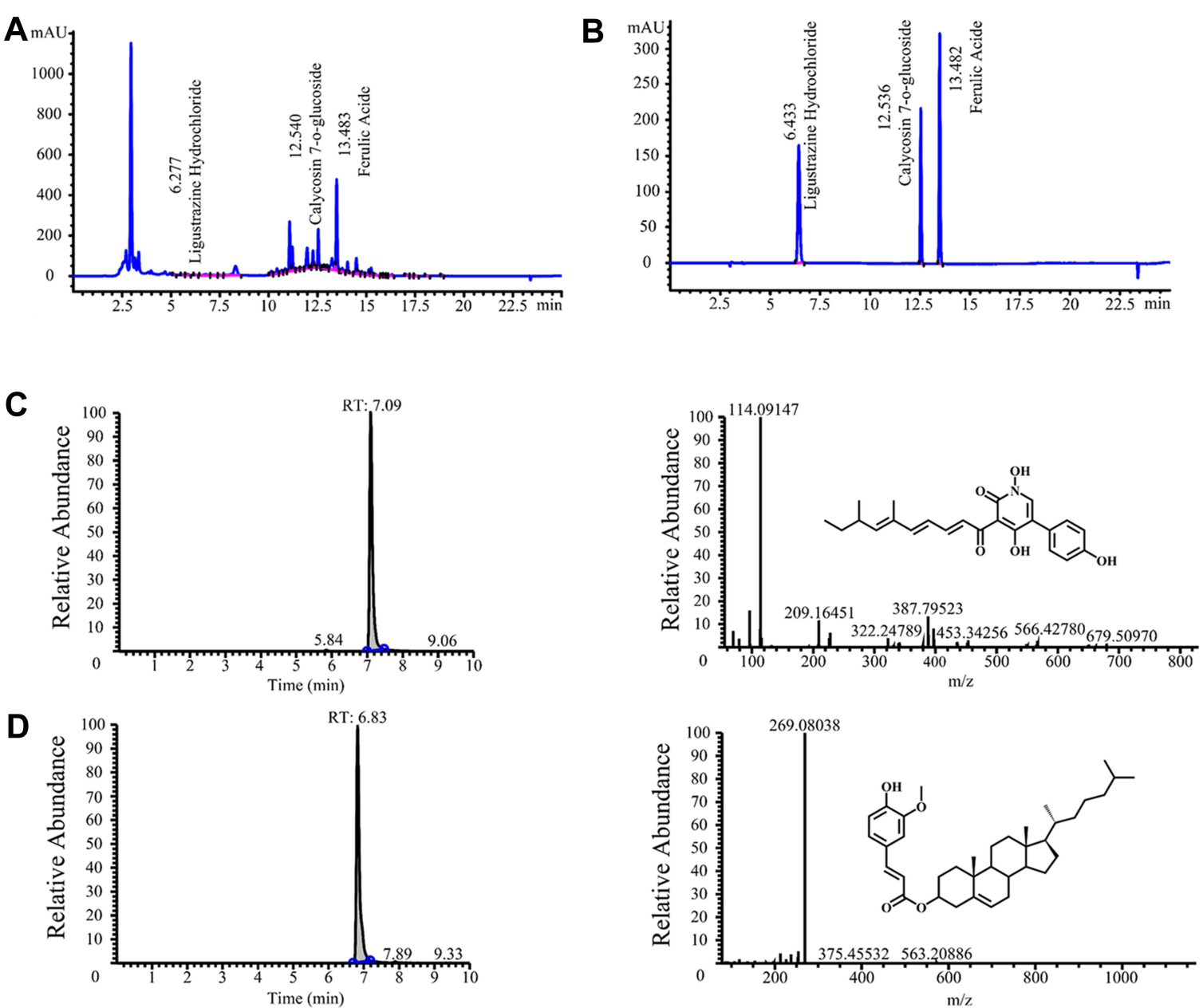

E
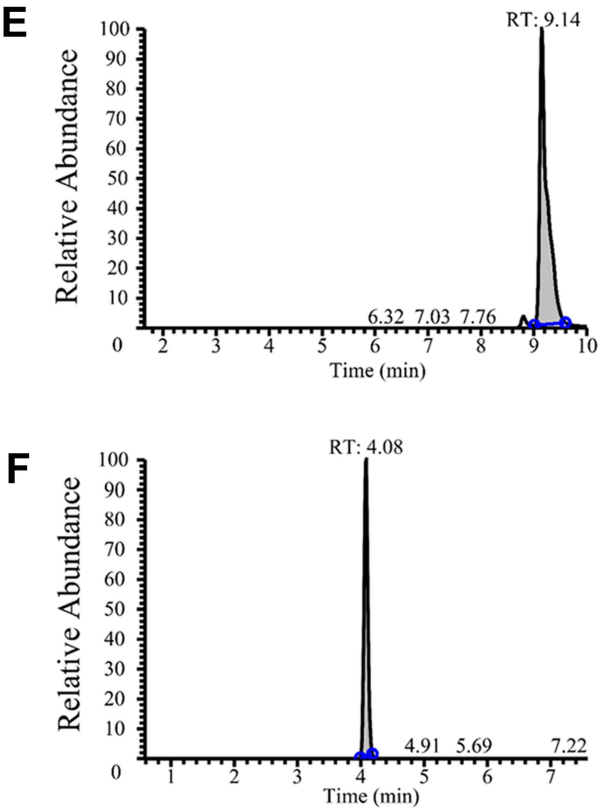
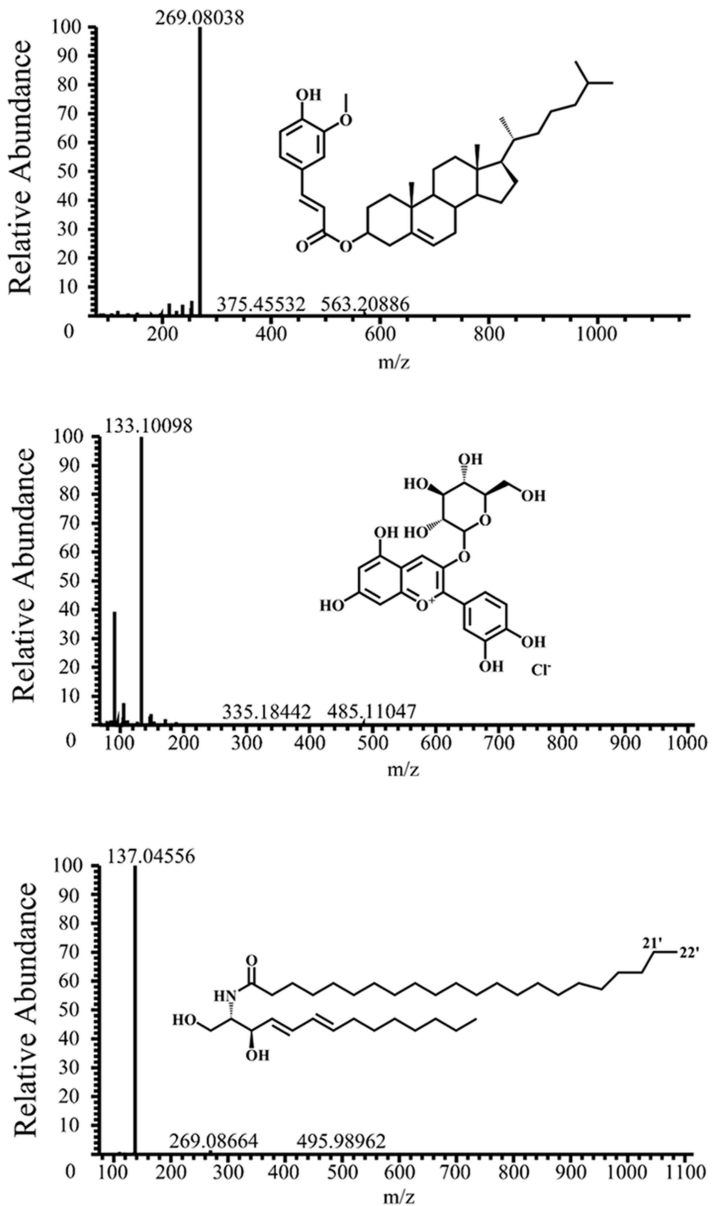

Figure 2 Chemical ingredients analysis of NTF. Representative ingredients of NTF (A) and standards (B). The chromatogram, mass spectrum and structural formula of four compounds: (C) Compound I; (D) Compound 2; (E) Compound 3; (F) Compound 4. 

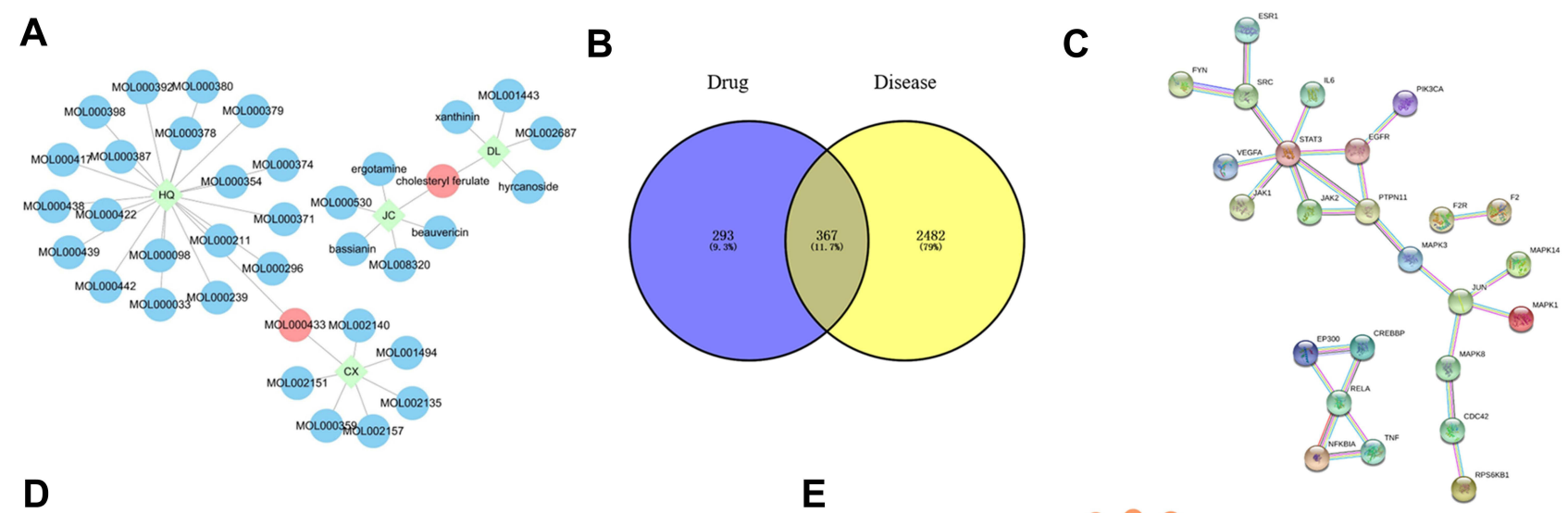

D

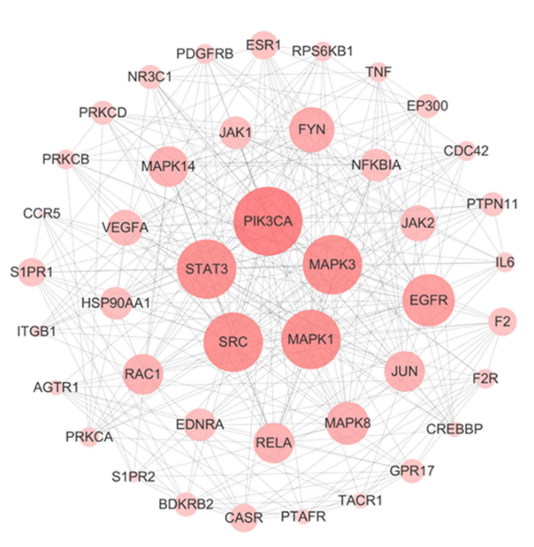

E

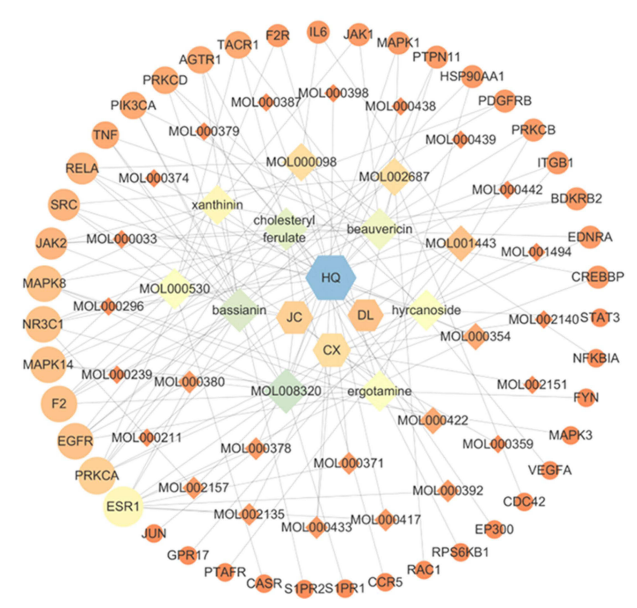

Figure 3 Prediction results of network pharmacology of NTF on ischemic stroke and CIRI. (A) The herb-compound network of NTF. Green nodes represent the herbs of NTF, orange nodes represent the central compounds of NTF, and blue nodes represent the other active compounds of NTF. (B) The venn diagram of the targets both in ischemic stroke targets whose color is yellow and NTF targets whose color is blue. (C) The results of topological screening for the PPI network ( $p>0.99$ ). (D) The graphical interactions network of the 43 key targets. The node color changes from yellow to red reflect the degree centrality changes from low to high. (E) The herb-compoundtarget network.

to lipid, phosphatidylinositol-mediated signaling, cellular response to growth factor stimulus and cellular response to organonitrogen compound. The molecular function terms $(p<0.05)$ were mainly related to the protein kinase binding, protein kinase activity, hormone receptor binding, insulin receptor substrate binding, protein tyrosine kinase activity, G protein-coupled peptide receptor activity, MAP kinase activity, transcription factor binding, $G$ protein-coupled receptor binding and integrin binding. Finally, the cellular component terms $(p<0.05)$ were mainly related to membrane raft, adherens junction and glutamatergic synapse.

To investigate the potential signaling pathways and functions of these key targets, the KEGG pathways for functional enrichment analysis were performed (Figure 4D). We conducted KEGG pathway enrichment analysis on 43 target genes and screened out 14 pathways based on the threshold of $p<0.05$ and targets-related signaling pathways. To analyze the pathways and their relationships to ischemic stroke and CIRI comprehensively, we selected the STAT3/PI3K/AKT signaling pathway to elucidate the potential mechanism of the protective effect of NTF on ischemic stroke and CIRI. Based on the results of these predicted molecular mechanisms and network analyses, we designed experiments to test our hypothesis in vivo.

\section{Molecular Docking Studies of Hit Compounds}

Judging from the topological analysis of the key targets network, PIK3CA had higher DC, BC and CC than other protein targets. According to the degree of herbcompound-target results, we selected the top 7 (degree $\geq 10$ ) and used the Glide module of the Schrodinger software to calculate the docking scores of the NTF, which expressed the energy of ligand-receptor binding that was inversely proportional to the binding affinity between ligand and receptor. According to the docking 
Table 2 Information on 43 Hub Targets

\begin{tabular}{|c|c|c|c|c|}
\hline Protein Name & Gene Symbol & DC & BC & CC \\
\hline Phosphatidylinositol-4,5-bisphosphate 3-kinase catalytic subunit alpha & PIK3CA & 29 & 0.144 & 0.764 \\
\hline Signal transducer and activator of transcription 3 & STAT3 & 25 & 0.073 & 0.712 \\
\hline Tyrosine-protein kinase SRC & SRC & 25 & 0.040 & 0.712 \\
\hline Mitogen-activated protein kinase I & MAPKI & 25 & 0.051 & 0.712 \\
\hline Mitogen-activated protein kinase 3 & MAPK3 & 25 & 0.0521 & 0.712 \\
\hline Epidermal growth factor receptor & EGFR & 22 & 0.049 & 0.677 \\
\hline Tyrosine-protein kinase Fyn & FYN & 19 & 0.021 & 0.646 \\
\hline Mitogen-activated protein kinase 8 & MAPK8 & 18 & 0.027 & 0.636 \\
\hline Transcription factor p 65 & RELA & 17 & 0.017 & 0.627 \\
\hline Transcription factor AP-I & JUN & 17 & 0.023 & 0.609 \\
\hline Mitogen-activated protein kinase 14 & MAPKI4 & 17 & 0.020 & 0.609 \\
\hline Ras-related C3 botulinum toxin substrate I & RACI & 17 & 0.014 & 0.627 \\
\hline Tyrosine-protein kinase JAK2 & JAK2 & 15 & 0.009 & 0.609 \\
\hline Vascular endothelial growth factor $\mathrm{A}$ & VEGFA & 15 & 0.019 & 0.600 \\
\hline NF-kappa-B inhibitor alpha & NFKBIA & 14 & 0.013 & 0.600 \\
\hline Tyrosine-protein kinase JAKI & JAKI & 14 & 0.007 & 0.600 \\
\hline Heat shock protein HSP 90 -alpha & HSP90AAI & 14 & 0.008 & 0.600 \\
\hline Endothelin-I receptor & EDNRA & 14 & 0.020 & 0.592 \\
\hline Thrombin & F2 & 13 & 0.012 & 0.568 \\
\hline Estrogen receptor & ESRI & 13 & 0.008 & 0.592 \\
\hline Sphingosine I-phosphate receptor I & SIPRI & 13 & 0.015 & 0.592 \\
\hline Extracellular calcium-sensing receptor & CASR & 13 & 0.009 & 0.545 \\
\hline Tyrosine-protein phosphatase non-receptor type II & PTPNII & 12 & 0.002 & 0.583 \\
\hline EIA binding protein $\mathrm{p} 300$ & EP300 & 12 & 0.004 & 0.532 \\
\hline Glucocorticoid receptor & $\mathrm{NR} 3 \mathrm{Cl}$ & 12 & 0.006 & 0.583 \\
\hline Protein kinase $C$ delta type & PRKCD & 12 & 0.003 & 0.583 \\
\hline B2 bradykinin receptor & BDKRB2 & 12 & 0.005 & 0.538 \\
\hline Uracil nucleotide/cysteinyl leukotriene receptor & GPRI7 & 12 & 0.005 & 0.538 \\
\hline Proteinase-activated receptor I & $\mathrm{F} 2 \mathrm{R}$ & 11 & 0.006 & 0.553 \\
\hline Interleukin-6 & IL6 & 11 & 0.004 & 0.553 \\
\hline Cell division control protein 42 & CDC42 & 11 & 0.005 & 0.568 \\
\hline Tumor necrosis factor & TNF & 11 & 0.003 & 0.525 \\
\hline Ribosomal protein S6 kinase beta-I & RPS6KBI & 11 & 0.006 & 0.575 \\
\hline Platelet-derived growth factor receptor beta & PDGFRB & 11 & 0.002 & 0.575 \\
\hline Protein kinase $C$ beta type & PRKCB & 11 & 0.003 & 0.538 \\
\hline Protein kinase $C$ alpha type & PRKCA & 11 & 0.003 & 0.538 \\
\hline CREB-binding protein & CREBBP & 10 & 0.002 & 0.512 \\
\hline Type-I angiotensin II receptor & AGTRI & 10 & 0.002 & 0.532 \\
\hline Platelet-activating factor receptor & PTAFR & 10 & 0.004 & 0.553 \\
\hline Substance-P receptor & TACRI & 10 & 0.002 & 0.532 \\
\hline Integrin beta-I & ITGBI & 9 & 0.001 & 0.553 \\
\hline Sphingosine I-phosphate receptor 2 & SIPR2 & 9 & 0.011 & 0.545 \\
\hline C-C chemokine receptor type 5 & CCR5 & 8 & 0.008 & 0.532 \\
\hline
\end{tabular}

score whose absolute value was greater than 5, we selected 3 common hub compounds, which were the hyrcanoside, bassianin and cholesteryl ferulate against the active sites of the identified protein targets PIK3CA. The total $2 \mathrm{D}$ and $3 \mathrm{D}$ interaction diagrams are respectively shown in Figure 5.

\section{NTF Decelerates the CIRI}

To further observe the effect of pretreatment with NTF extract on the degree of injury in rats, a neurological scores system was first established to evaluate scathing degree based on behavioral changes in rats after $24 \mathrm{~h}$ of reperfusion. TTC staining was used to evaluate cerebral 


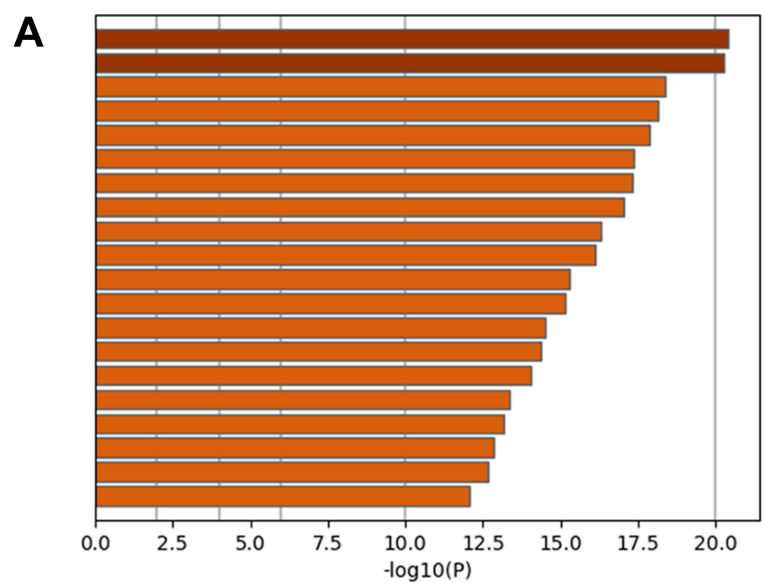

B

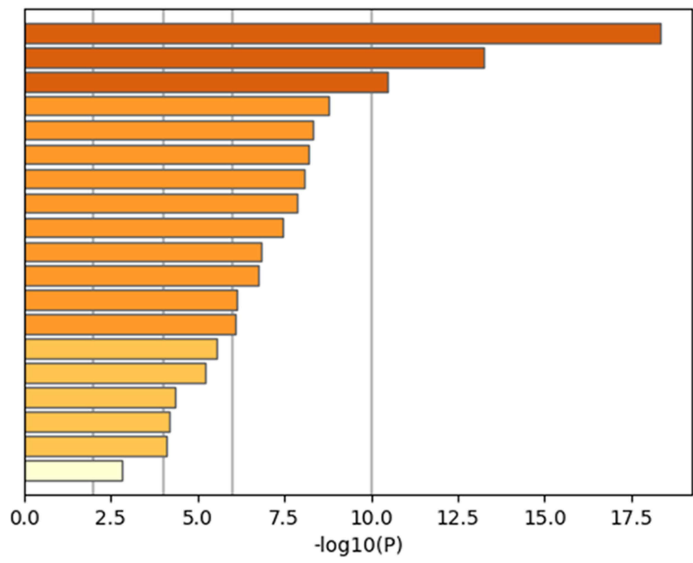

C

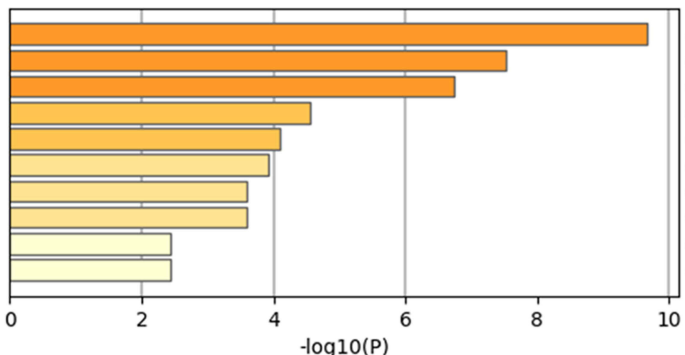

$-\log 10(P)$

D

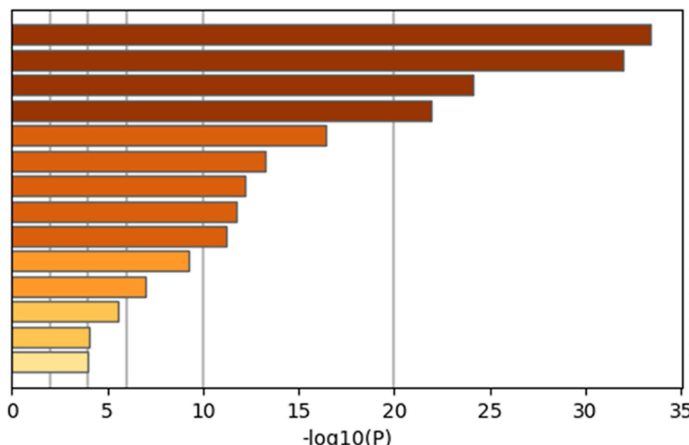

GO:0004879: nuclear receptor activity

hsa04071: Sphingolipid signaling pathway
GO:0045121: membrane raft

GO:0005912: adherens junction

GO:0098978: glutamatergic synapse

GO:0031983: vesicle lumen

GO:0098552: side of membrane

GO:0030425: dendrite

GO:0031256: leading edge membrane

GO:0048471: perinuclear region of cytoplasm

GO:0005819: spindle

GO:0005667: transcription factor complex

GO:0042330: taxis

signaling pathway

. positive regulation of kin

:0071363: cellular response to growth factor stimulus

:0051090: rellular response to organonitrogen compound

GO:2000377: regulation of reactive oxygen species metabolic process

: cation homeostasis

(lifferentiation

10

GO:0001819: positive regulation of cytokine production

GO:0019901: protein kinase binding

GO:0004672: protein kinase activity

insulin receptor substrate binding

prinase activity

7. MAP kinase activity

GO:0005178: integrin binding

:

GO:0019902: phosphatase binding

tectivity

26.

O:0048156: tau protein binding$$
\text { . }
$$

(1)

hsa05200: Pathways in cancer

hsa04933: AGE-RAGE signaling pathway in diabetic complications hsa05205: Proteoglycans in cancer

hsa04931: Insulin resistance

hsa05418: Fluid shear stress and atherosclerosis

hsa04020: Calcium signaling pathway

hsa05162: Measles

hsa04750: Inflammatory mediator regulation of TRP channels

hsa05221: Acute myeloid leukemia

hsa05321: Inflammatory bowel disease (IBD)

hsa05130: Pathogenic Escherichia coli infection

hsa04144: Endocytosis

hsa05014: Amyotrophic lateral sclerosis (ALS)

Figure 4 Functional analysis of NTF. GO enrichment of related genes of (A) biological process, (B) molecular functions, (C) cellular components and (D) KEGG pathway enrichment analysis for 43 key targets. 
A

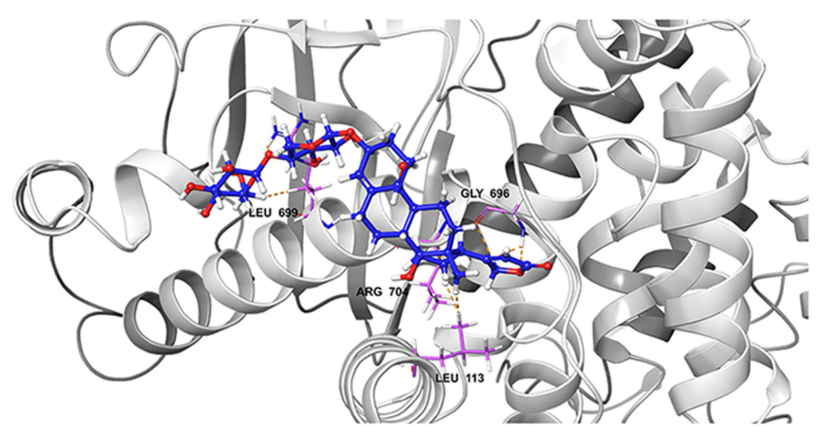

B

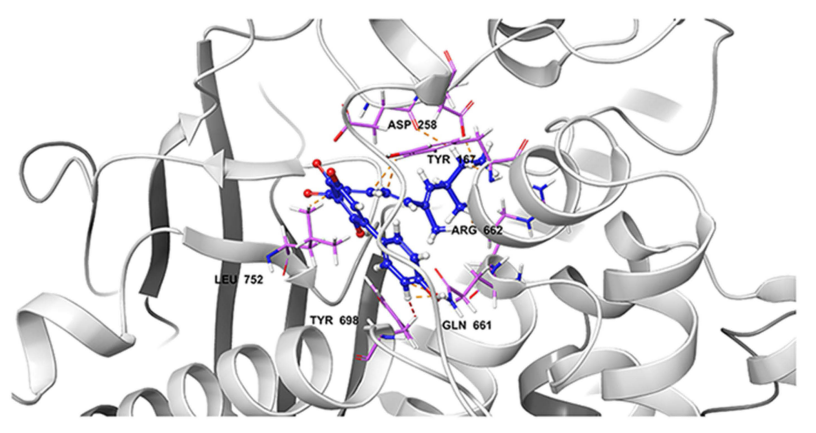

C

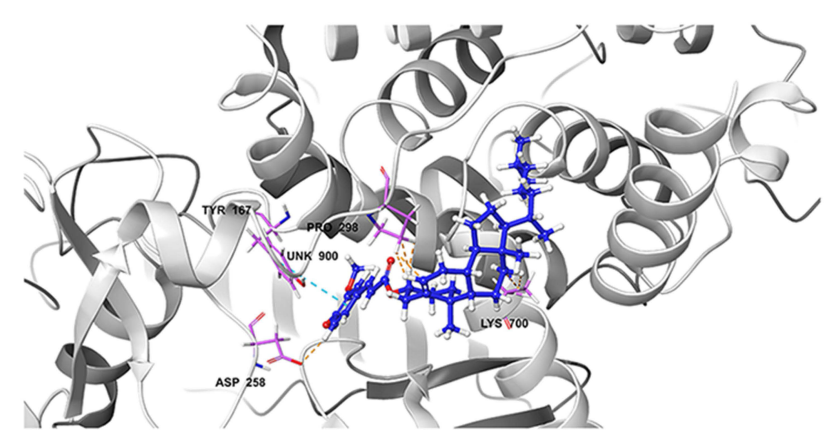

a

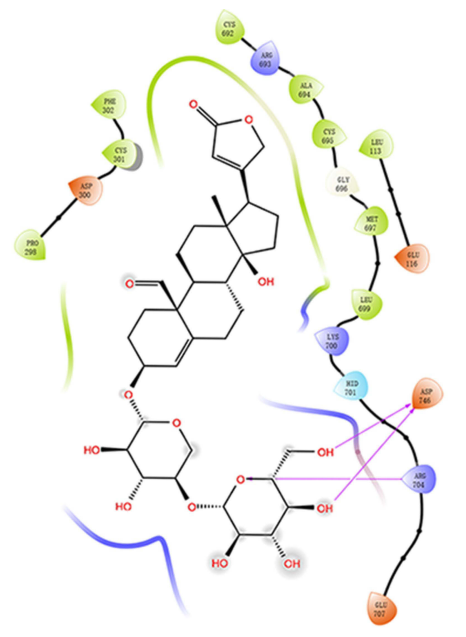

b

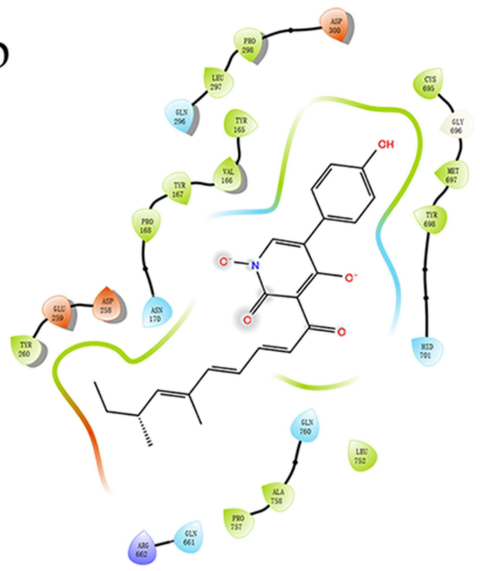

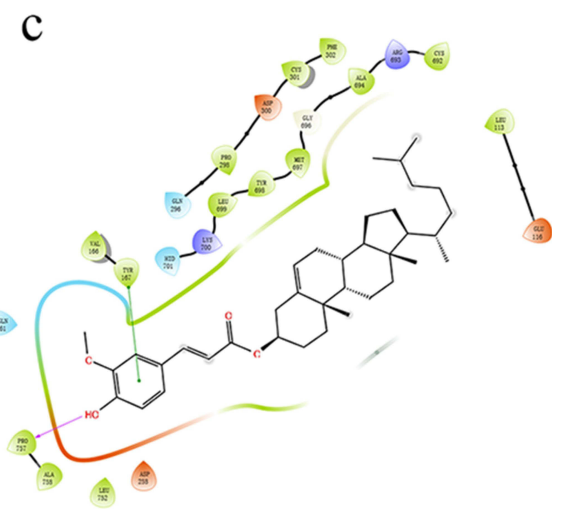

Figure 5 3D and 2D interaction diagrams of hyrcanoside (Aa), bassianin (Bb) and cholesteryl ferulate (Cc) in the active site of PIK3CA (PDB ID 4TUU). 
infarct volume. The results showed that the neurological score and infarct volume of the model group were significantly higher than those of the sham group $(p<0.01)$. Compared with the model group, NTF could significantly reduce the neurological score $(p<0.01)$ and infarct volume $(p<0.05)$ of rats after CIRI (Figure 6). Then the morphology of brain nerve cells and the density of dendritic spines in ischemic cortex and hippocampus of rats were observed after the Nissl staining and Golgi staining. In model group, multiple neurons were destroyed, and damages were reduced dramatically by NTF extracts pretreatment. In the model group, the spines density in cortex and hippocampus were significantly downregulated compared to the sham group $(p<0.01)$. Compared with the model group, the spines density was significantly increased in cortex and hippocampus in the NTF group $(p<0.05)$ (Figure 7). In the model group, numbers of Nissl bodies in cortex and hippocampus were significantly decreased compared to the sham group $(p<0.01)$. Compared with the model group, the number of Nissl bodies were significantly increased in cortex $(p<0.01)$ and hippocampus $(p<0.05)$ in the NTF group (Figure 8). TUNEL staining identifies apoptotic neurons. As shown in Figure 9, there were many TUNEL- positive neurons in ischemic cortex and hippocampus of CIRI rats in the model group $(p<0.01)$ compared with the sham group, and fewer TUNEL-positive neurons in the NTF group compared with the model group $(p<0.01)$. The results indicated that pretreatment of NTF might exert a protective effect against apoptosis in rats subjected to CIRI.

\section{Verification of Critical Proteins in NTF on CIRI by Immunofluorescence and Western Blotting}

To explore the intracellular mechanisms of NTF extract on STAT3/PI3K/AKT signaling pathway in CIRI, the expressions of p-STAT3, PI3K, p-AKT, AKT, Bcl-2 and Bax in CIRI brain tissues were evaluated. The immunofluorescence staining results are shown in Figures 10-14. Compared with the sham group, the levels of p-STAT3, $\mathrm{PI} 3 \mathrm{~K}, \mathrm{p}-\mathrm{AKT}$ and Bcl-2 in cortex and hippocampus were obviously downregulated in the model group $(p<0.01)$. The levels of Bax in cortex and hippocampus were obviously upregulated in the model group $(p<0.01)$. Compared with the model group, the levels of Bax were
A

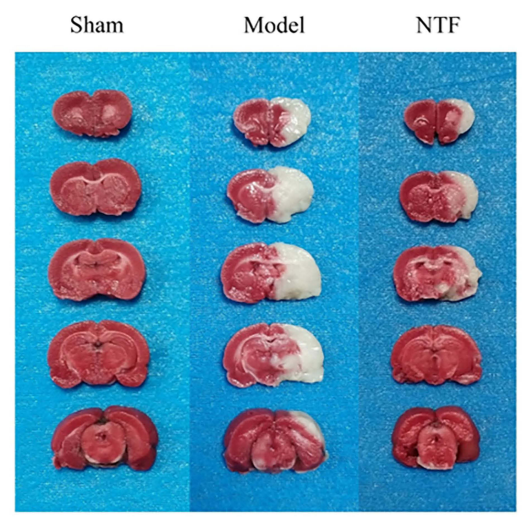

B

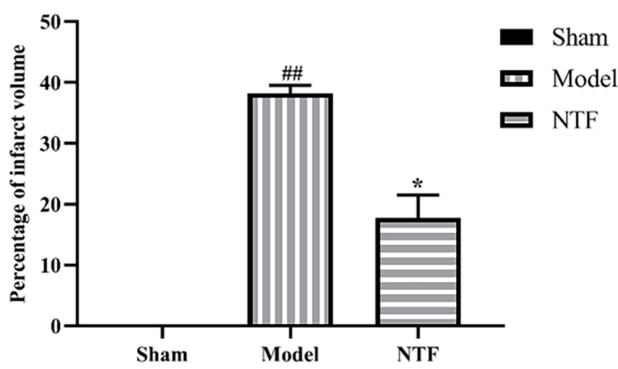

C

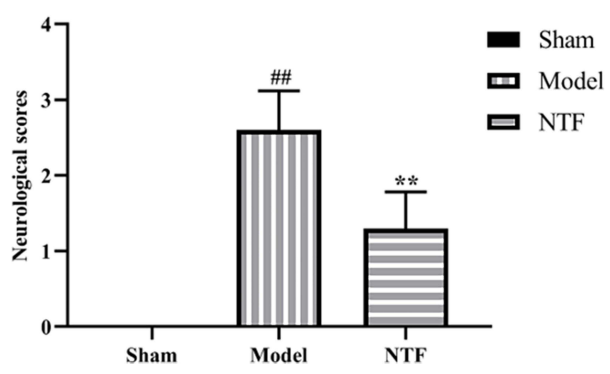

Figure 6 Pretreatment of NTF 7 days prior to CIRI reduced infarct volume and ameliorated neurological deficit in rats 24 h after reperfusion. (A) Representative TTCstained photos of the cerebral infarct coronal sections. (B) Percentage of infarct volume. (C) Neurological scores. All data were presented as mean \pm SD. ${ }^{\#} p<0.0$ I versus sham group; $* * p<0.01,{ }^{*} p<0.05$ versus model group, respectively. 

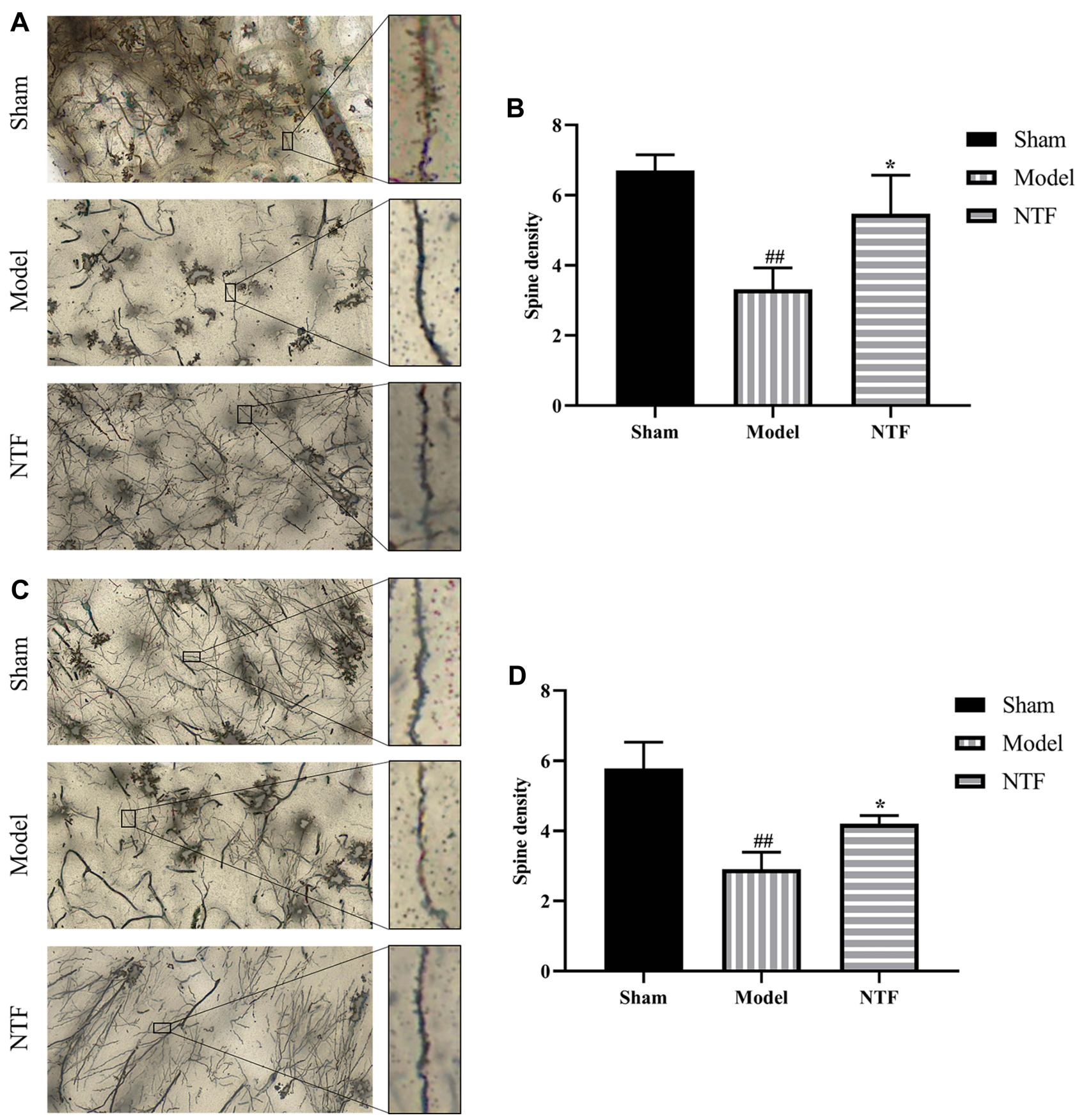

Figure 7 Pretreatment of NTF 7 days prior to CIRI increased the spines density in rats $24 \mathrm{~h}$ after reperfusion. (A) Representative Golgi-stained photos of the dendritic spines in ischemic cortex sections magnified 400 times. (B) Spines density in ischemic cortex. (C) Representative Golgi-stained photos of the dendritic spines in ischemic

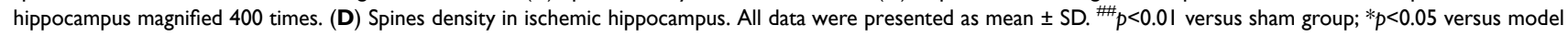
group, respectively.

obviously downregulated in cortex $(p<0.01)$ and hippocampus $(p<0.05)$ in the NTF group. The levels of p-STAT3 in cortex were upregulated in the NTF group $(p<0.01)$. And the levels of PI3K, p-AKT and Bcl-2 in cortex were obviously upregulated in the NTF group $(p<0.05)$. In hippocampus, the levels of PI3K and Bcl-2 were upregulated in the NTF group $(p<0.05)$, as well as the levels of p-AKT $(p<0.01)$. The levels of p-STAT3 were raised in the NTF group without significant difference $(p>0.05)$. As shown in Figure 15, the Western blotting showed the protein expressions of Bcl-2, Bax, p-STAT3, PI3K, p-AKT, AKT. Compared with the sham group, Bcl- 

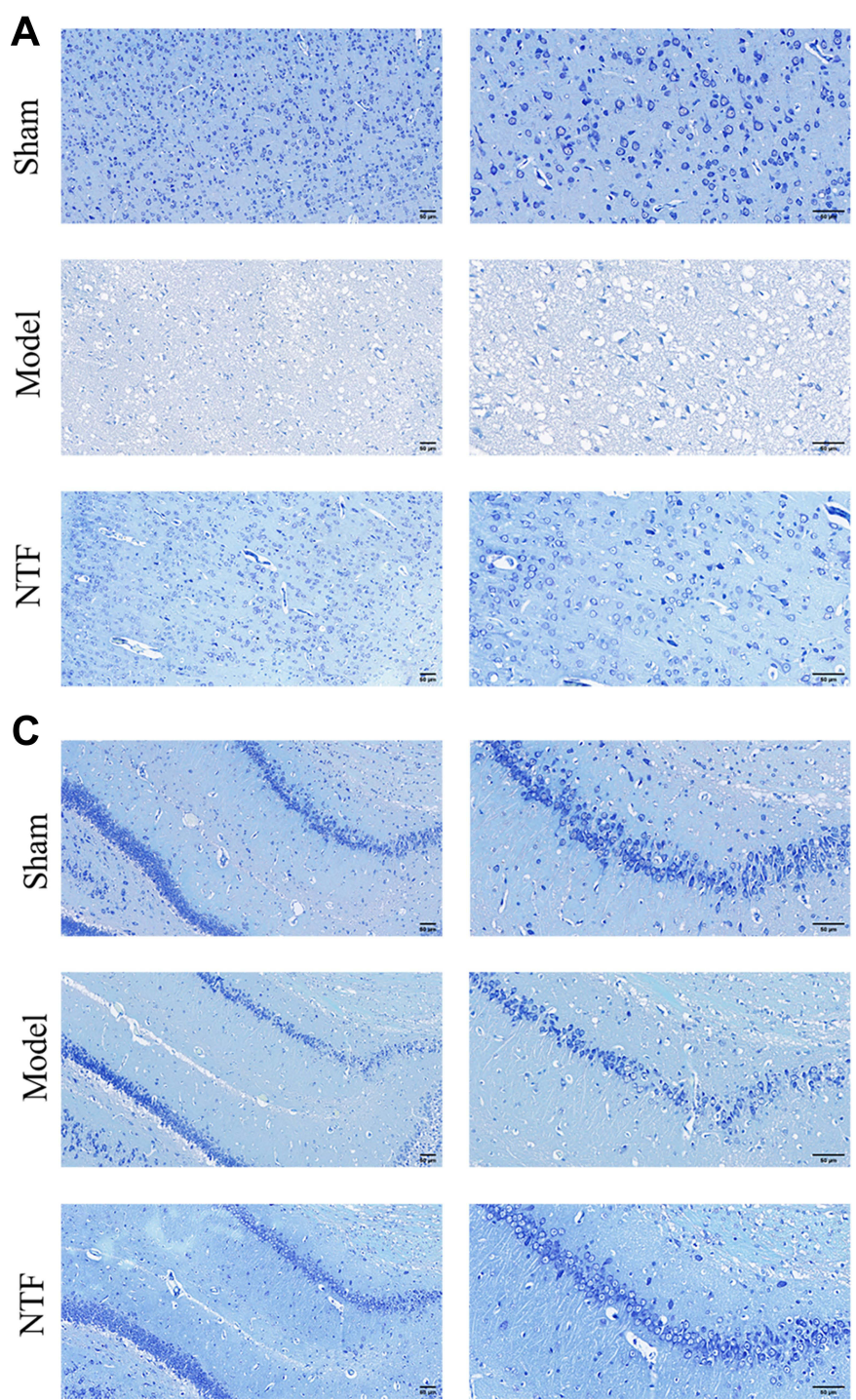
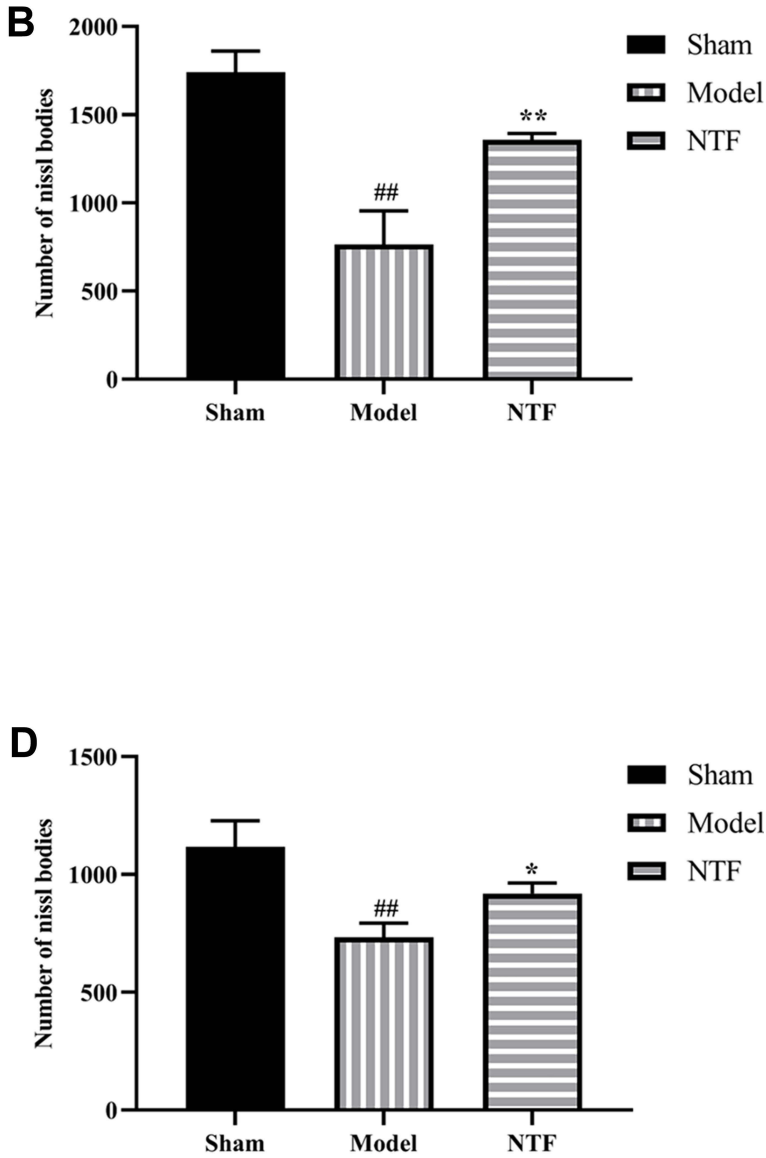

Figure 8 Pretreatment of NTF 7 days prior to CIRI increased the neurons activity of the ischemic cortex and hippocampus in rats $24 \mathrm{~h}$ after reperfusion. (A) Representative images magnified 200 times and 400 times in ischemic cortex sections. (B) Quantitatively analyzed Nissl bodies in each group after $24 \mathrm{~h}$ of reperfusion in ischemic cortex. (C) Representative images magnified 200 times and 400 times in ischemic hippocampus sections. (D) Quantitatively analyzed Nissl bodies in each group after $24 \mathrm{~h}$ of reperfusion in ischemic hippocampus. All data were presented as mean $\pm S D\left(n=18\right.$ rats in each group). ${ }^{\# \#} p<0.01$ versus sham group; ${ }^{* *} p<0.01$, ${ }^{*} p<0.05$ versus model group, respectively.

2/Bax ratio and p-STAT3 in the model group were significantly decreased in cortex and hippocampus $(p<0.01)$. The levels of PI3K were obviously downregulated in cortex $(p<0.05)$ and hippocampus $(p<0.01)$. The $\mathrm{p}$-AKT/AKT ratio in the model group were significantly decreased in cortex and hippocampus $(p<0.05)$. Compared with the model group, the levels of Bcl-2/Bax, p-STAT3, p-AKT/ AKT in the NTF group were obviously raised in cortex and hippocampus $(p<0.05)$. The levels of PI3K were obviously upregulated in hippocampus $(p<0.05)$. In cortex, the levels of PI3K were upregulated without statistical differences $(p>0.05)$. These results suggested that pretreatment of NTF could protect the brain against ischemic stroke and CIRI via STAT3/PI3K/AKT signaling pathway.

\section{Discussion}

Successful recanalization of arterial blood flow is the primary therapeutic option after the onset of ischemic stroke. However, complications may occur after revascularization, and CIRI is one of the most serious complications. CIRI is defined as a biochemical cascade reaction causing the deteriorating effects in ischemic brain tissue, whose changes of function and structure 

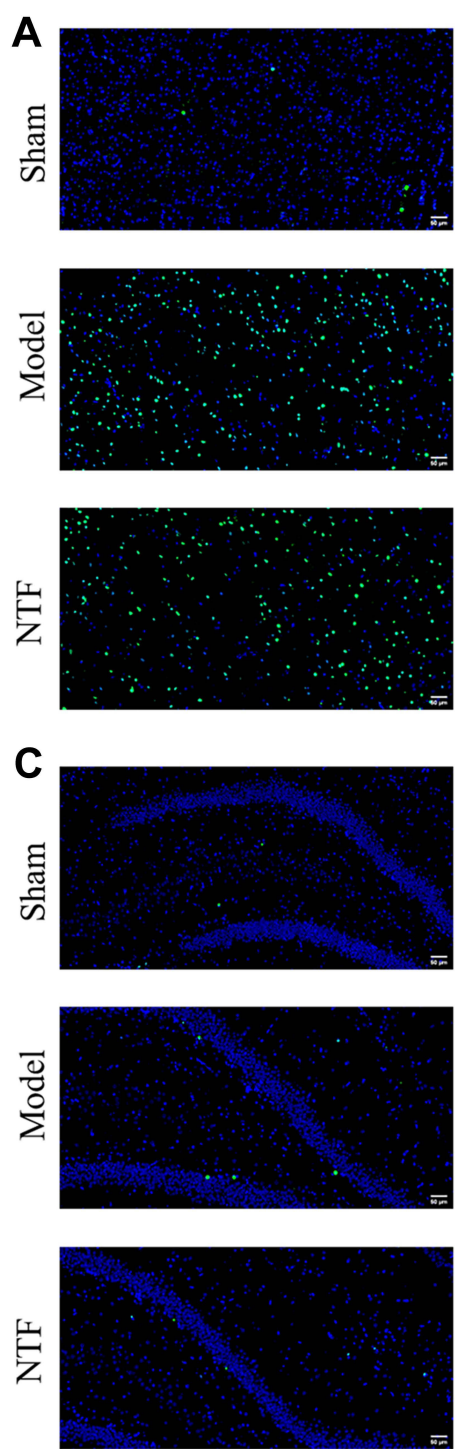
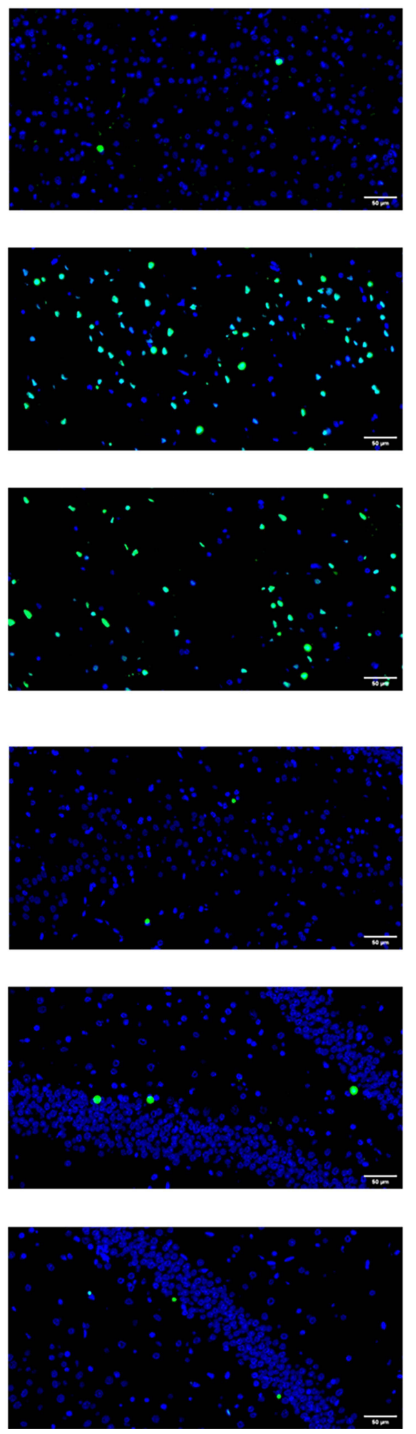
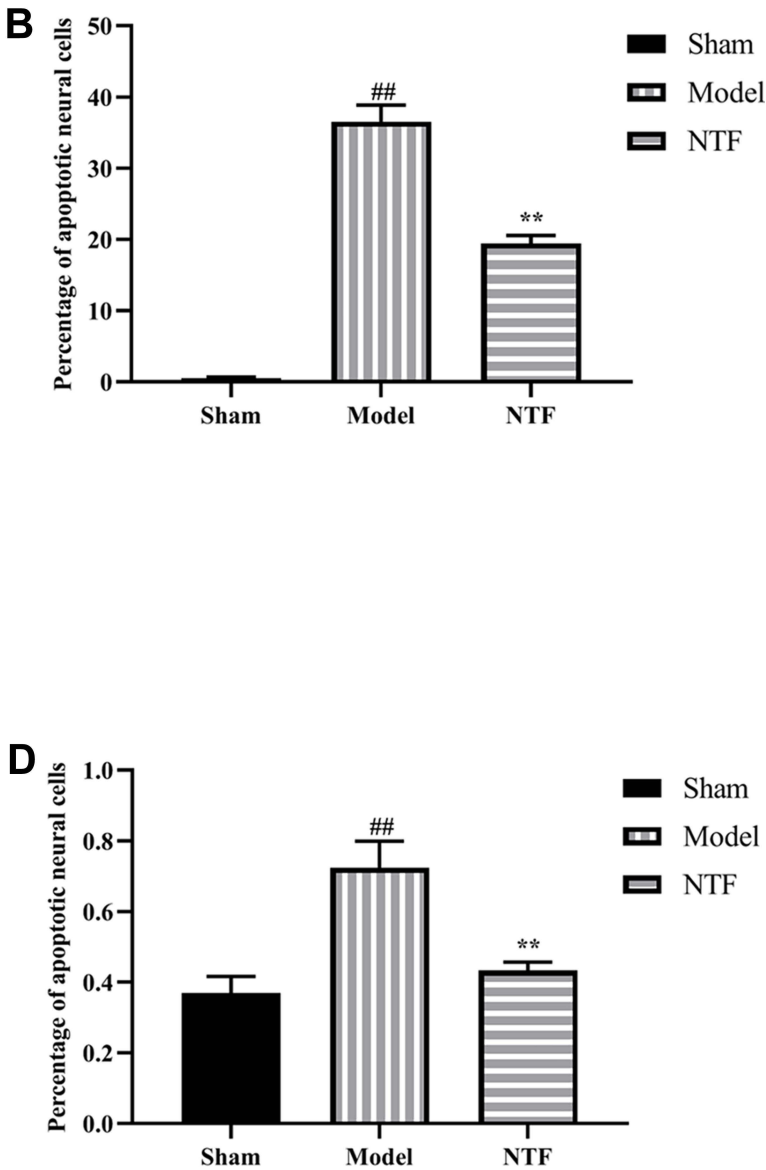

Figure 9 Pretreatment of NTF 7 days prior to CIRI reduced the neuronal apoptosis of the ischemic cortex and hippocampus in rats $24 \mathrm{~h}$ after reperfusion. (A) Representative images magnified 200 times and 400 times in ischemic cortex sections. (B) The apoptotic index indicates the percentage of TUNEL-positive cells in each group after $24 \mathrm{~h}$ of reperfusion in ischemic cortex. (C) Representative images magnified 200 times and 400 times in ischemic hippocampus sections. (D) The apoptotic index indicates the percentage of TUNEL-positive cells in each group after $24 \mathrm{~h}$ of reperfusion in ischemic hippocampus. All data were presented as mean \pm SD. ${ }^{\#} p<0.0 \mathrm{I}$ versus sham group; $* * p<0.01$ versus model group, respectively.

become conspicuous during the restoration of the blood flow after a period of ischemic stroke, which is a common and inevitable problem after revascularization therapy. $^{38}$ The damage of tissues caused by CIRI is divided into two parts: ischemia injury and reperfusion injury. The main factor for ischemia injury is cell energy depletion, and for reperfusion injury, the main factors are interplay of oxidative and microcirculatory stress, along with inflammation and apoptosis. ${ }^{39}$ After the reperfusion stage, red blood cells restore blood flow to provide oxygen to ischemic tissue. Moreover, a lower concentration of anti-oxidative agents in ischemic cells increases local inflammation and ROS production, leading to secondary injury. ${ }^{40,41}$ Apoptosis is also a crucial mechanism of secondary damage in brain tissue after CIRI, the overproduction of ROS and the translocation of Bax (pro-apoptotic protein) trigger the intrinsic apoptotic pathway. ${ }^{42,43}$ Delays to reperfusion, incomplete recanalization, hemorrhagic transformation, and secondary injury after reperfusion are some of the limitations of intravenous thrombolysis. ${ }^{44}$ The subsequent injury induced by CIRI is a critical condition for which physicians need to preserve organ and neurogenic function. As new facts became known, compound Chinese 
A
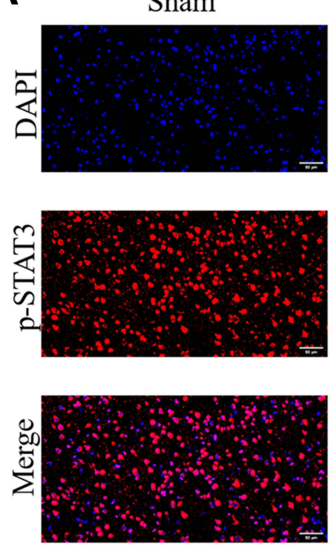

C
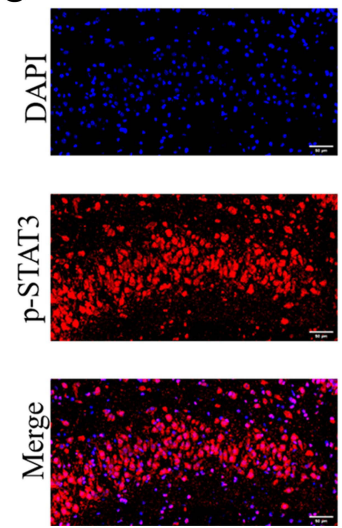
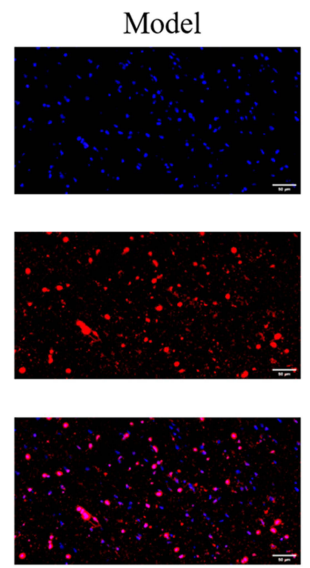

Model
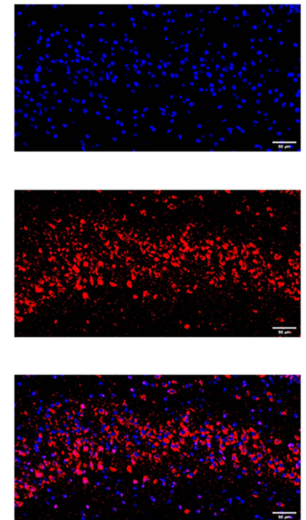
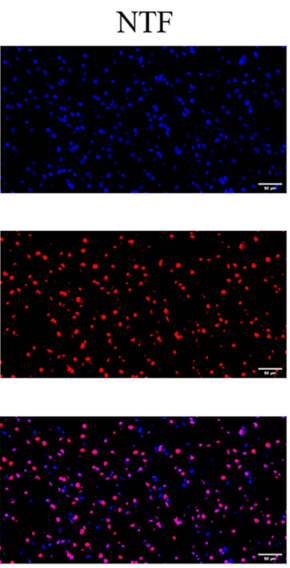

NTF
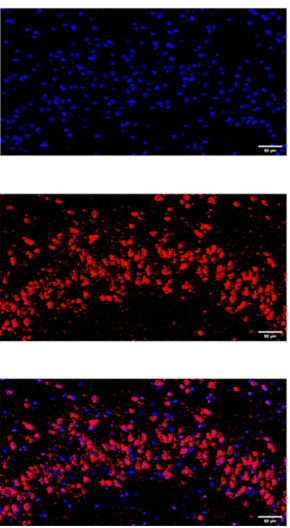

B

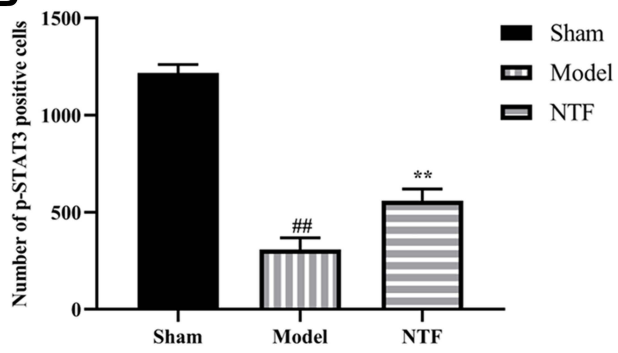

D

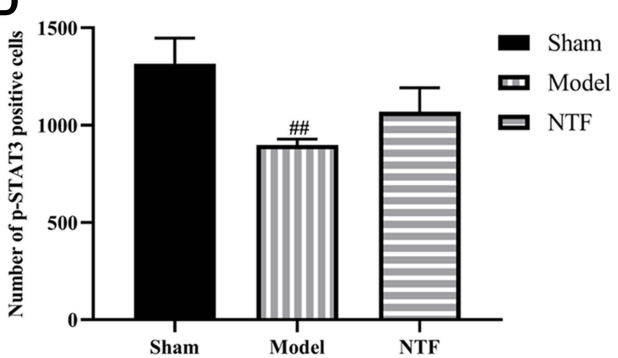

Figure 10 Effects of NTF 7 days prior to CIRI on expression of p-STAT3 of the ischemic cortex and hippocampus in rats $24 \mathrm{~h}$ after reperfusion. (A) Representative images magnified 400 times in ischemic cortex sections examined with specific antibody against p-STAT3 (red); nuclei were stained with DAPI (blue). (B) The numbers of p-STAT3 positive cells in each group after $24 \mathrm{~h}$ of reperfusion in ischemic cortex. (C) Representative images magnified 400 times in ischemic hippocampus sections. (D) The numbers of p-STAT3 positive cells in each group after $24 \mathrm{~h}$ of reperfusion in ischemic hippocampus. All data were presented as mean \pm SD. ${ }^{\# \#} p<0.0 \mathrm{I}$ versus sham group; ${ }^{* *} p<0.0 \mathrm{I}$ versus model group, respectively.

medicine usually contains a large number of chemical components, which may work together to achieve a therapeutic effect of CIRI. ${ }^{45}$ Our previous research reports pointed out that NTF extract had a neuroprotective effect. ${ }^{20-23}$ Therefore, it is necessary to explore the mechanisms underlying the protective effects of NTF as an alternative or complementary medicine to prevent or alleviate CIRI in ischemic stroke patients.

In recent years, network pharmacology strategy has been successfully applied to analyze TCM prescriptions, optimize prescriptions, develop new medicines, screen the active ingredients of TCM, and research drugsymptoms, ${ }^{46}$ which is useful to explore the mechanisms underlying NTF in ischemic stroke and CIRI. Through active compounds screening, drug targeting and pathway enrichment analysis, NTF network pharmacology analysis identified 4 kinds of traditional Chinese medicine, 38 compounds and 43 hub targets in the present research. Meanwhile, the molecular docking results showed that the key active compounds in NTF had good binding affinity with 3 hub targets. Judging from the topological analysis of the key target-compound network and docking score of the molecular docking method, we selected 3 hub compounds, which were bassianin, cholesteryl ferulate, and hyrcanoside. It has been reported that astragaloside IV and calycosin-7-o-glucoside are the representative components of Radix Astragali, which may exert neuroprotective effects in CIRI mainly through their anti-oxidant, anti-inflammatory, anticoagulant, and anti-apoptotic actions. ${ }^{4,48}$ Ligustrazine and ferulic acid are the main active ingredients of Rhizoma Ligustici Chuanxiong. Previous studies have indicated that ligustrazine can protect against I/R injury via activating PI3K/AKT pathway based on its antiinflammatory and neuro-protective effects and ferulic 

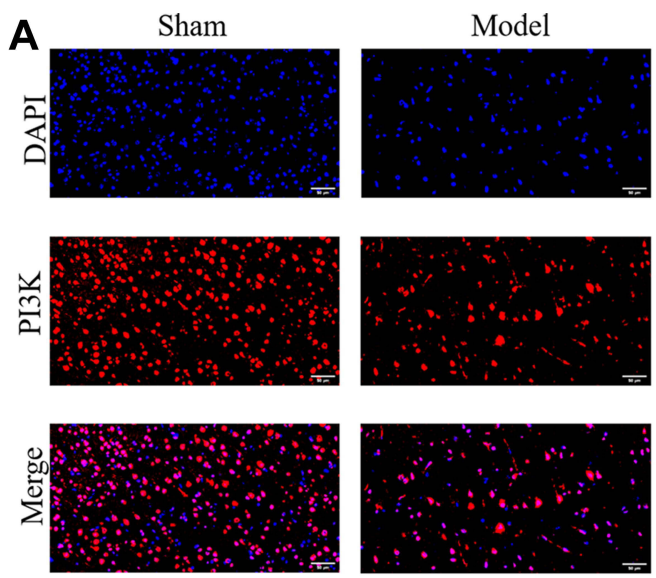

C

Sham
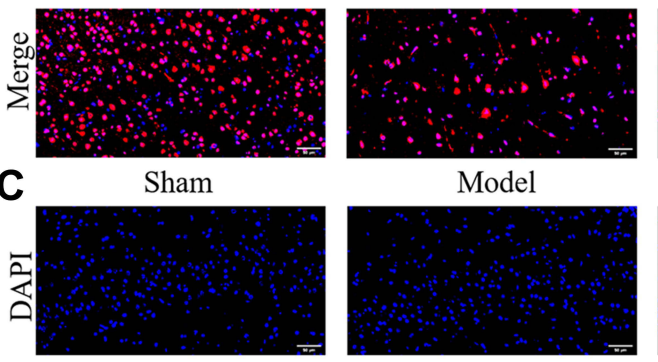

Model
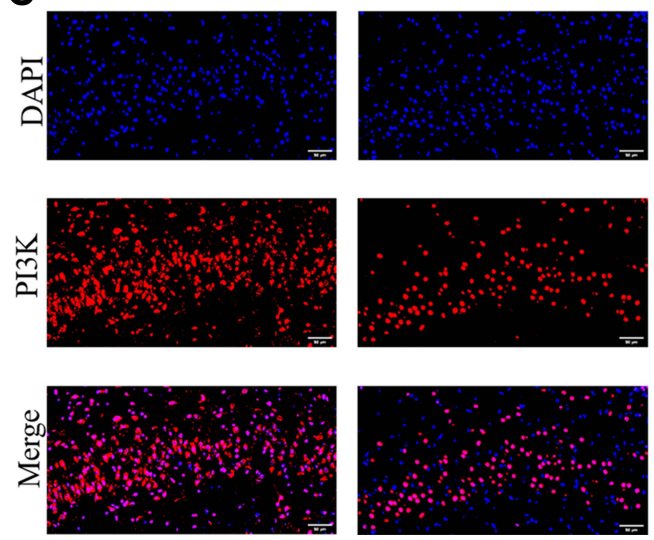
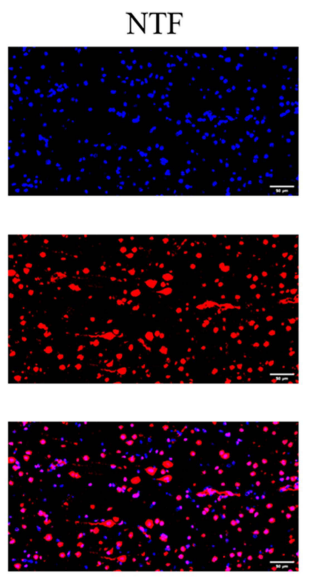

NTF
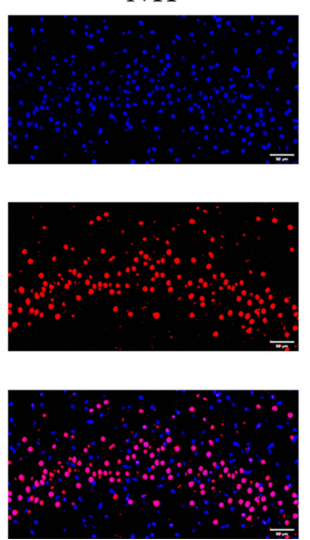

B

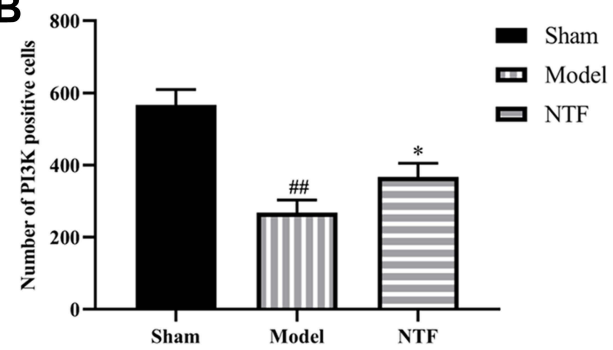

D

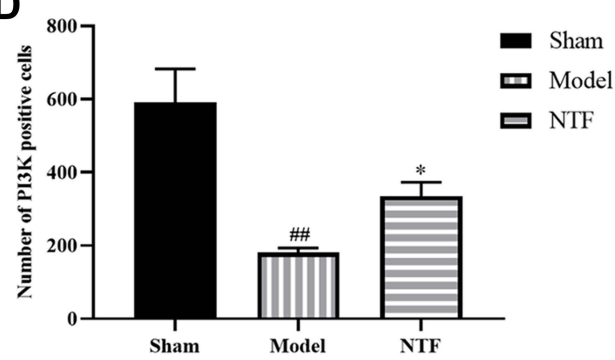

Figure II Effects of NTF 7 days prior to CIRI on expression of PI3K of the ischemic cortex and hippocampus in rats $24 \mathrm{~h}$ after reperfusion. (A) Representative images magnified 400 times in ischemic cortex sections examined with specific antibody against PI3K (red); nuclei were stained with DAPI (blue). (B) The numbers of PI3K positive cells in each group after $24 \mathrm{~h}$ of reperfusion in ischemic cortex. (C) Representative images magnified 400 times in ischemic hippocampus sections. (D) The numbers of PI3K positive cells in each group after $24 \mathrm{~h}$ of reperfusion in ischemic hippocampus. All data were presented as mean \pm SD. ${ }^{\# \#} p<0.01$ versus sham group; ${ }^{*} p<0.05$ versus model group, respectively.

acid acts as a neuroprotective function against cerebral ischemia through regulating p70S6 kinase expression and S6 phosphorylation which are downstream targets of AKT. ${ }^{49,50}$ Previous reports have shown that Pheretima could exert anti-inflammatory effects by reducing the production of inflammatory mediators such as NO, PGE2, TNF- $\alpha$ and various inflammatory cytokines. ${ }^{51}$ There is a research revealed that Pheretima may have anti-coagulant and anti-thrombotic functions through regulating DPf3, which is a group of antithrombotic functional proteins purified from Pheretima. ${ }^{52}$ Moreover, Bombyx Batryticatus has been reported to exhibit pharmacological activity on the nervous system, such as anticonvulsant and antiepileptic effects, hypnotic effects, and neurotrophic effects and so on. ${ }^{17}$ It has been also reported that $(4 \mathrm{E}, 6 \mathrm{E}, 2 \mathrm{~S}, 3 \mathrm{R})-2-\mathrm{N}$-docosanoyl4,6-tetradecasphingadienine is the potential structure in Bombyx Batryticatus with a neurite outgrowth promoting activity. ${ }^{53}$ Overall, the above findings suggested that the multiple-compounds of NTF may collectively exert beneficial effects against CIRI. And the HPLC and HRMS results in our study also showed the existence and contents of bassianin, cholesteryl ferulate, hyrcanoside, (4E,6E,2S,3R)-2-N-docosanoyl-4,6-tetradecasphingadienine, ligustrazine hydrochloride, calycosin 7-o-glucoside, and ferulic acid in NTF, which provided the pharmacological evidence for NTF to be used as an agent in the treatment of CIRI.

In this study, numerous targets and pathways were identified to be associated with multiple compounds from different herbs in NTF, which revealed the synergistic property of the compounds in the NTF for the treatment of ischemic stroke and CIRI. It was mainly based on the effective clustering of functional genes of GO and KEGG pathways, ${ }^{54,55}$ clustering functional genes into different biological processes, and it also 


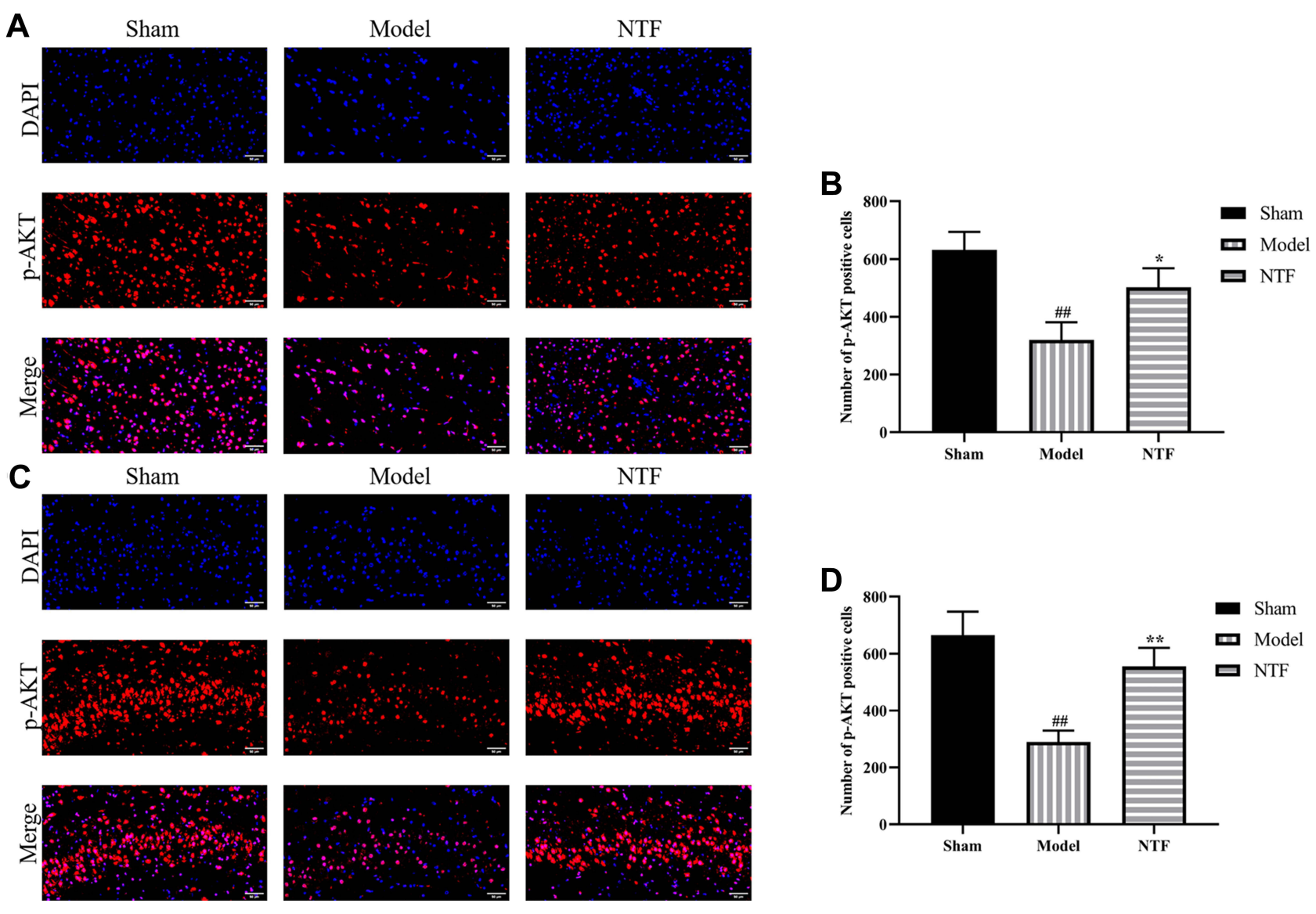

Figure 12 Effects of NTF extracts 7 days prior to CIRI on expression of p-AKT of the ischemic cortex and hippocampus in rats $24 \mathrm{~h}$ after reperfusion. (A) Representative images magnified 400 times in ischemic cortex sections examined with specific antibody against p-AKT (red); nuclei were stained with DAPI (blue). (B) The numbers of $\mathrm{P}$-AKT positive cells in each group after $24 \mathrm{~h}$ of reperfusion in ischemic cortex. (C) Representative images magnified 400 times in ischemic hippocampus sections. (D) The numbers of $\mathrm{p}$-AKT positive cells in each group after $24 \mathrm{~h}$ of reperfusion in ischemic hippocampus. All data were presented as mean \pm SD. ${ }^{\#} p<0.0 \mathrm{l}$ versus sham group; $* * p<0.01, * p<0.05$ versus model group, respectively.

helped to solve these problems related to the underlying therapeutic mechanism of NTF's effects on ischemic stroke and CIRI. Notably, it has shown that the STAT3/PI3K/AKT signaling pathway related to hub targets are considered to be potentially correlated with ischemic stroke and CIRI. The STAT3/PI3K/AKT signaling pathway is reported to play important roles in the treatment of ischemic stroke and CIRI through cell growth and differentiation regulation, ${ }^{56,57}$ apoptosis, ${ }^{58,59}$ restoration and survival of ischemic nerve cells, ${ }^{60,61}$ neuro-inflammation, ${ }^{62}$ angiogenesis ${ }^{63}$ and so on. In a word, the above research revealed that NTF could protect the brain tissue against ischemic stroke and CIRI via the STAT3/PI3K/AKT signaling pathway.

The results of this study showed that the neurological function of CIRI rats was improved, the ischemic infarct volume was reduced, and the dendritic spine density and survival of neurons were increased after pretreatment of NTF for 7 days, which indicated that NTF pre-administration had an effective neuroprotective effect on CIRI. The cells of the ischemic core area experience a sudden reduction of blood flow, within just several minutes after ischemic attack with irreversible injury and subsequent cell death, and the main form of cell death is apoptosis. ${ }^{59}$ CIRI triggers multiple cell apoptotic pathways. $^{64}$ The JAK/STAT signaling pathway is the main signal mechanism for a wide array of cytokines and growth factors in mammals and it is a crucial player in promoting neurogenesis, which facilitates neuronal differentiation and synaptic plasticity. ${ }^{65,66}$ There are seven members of the STAT family in the cytoplasm, namely STAT1, STAT2, STAT3, STAT4, STAT5A, STAT5B and STAT6, ${ }^{67,68}$ which have been reported to be involved in cell oxidative stress, apoptosis, proliferation, differentiation, 


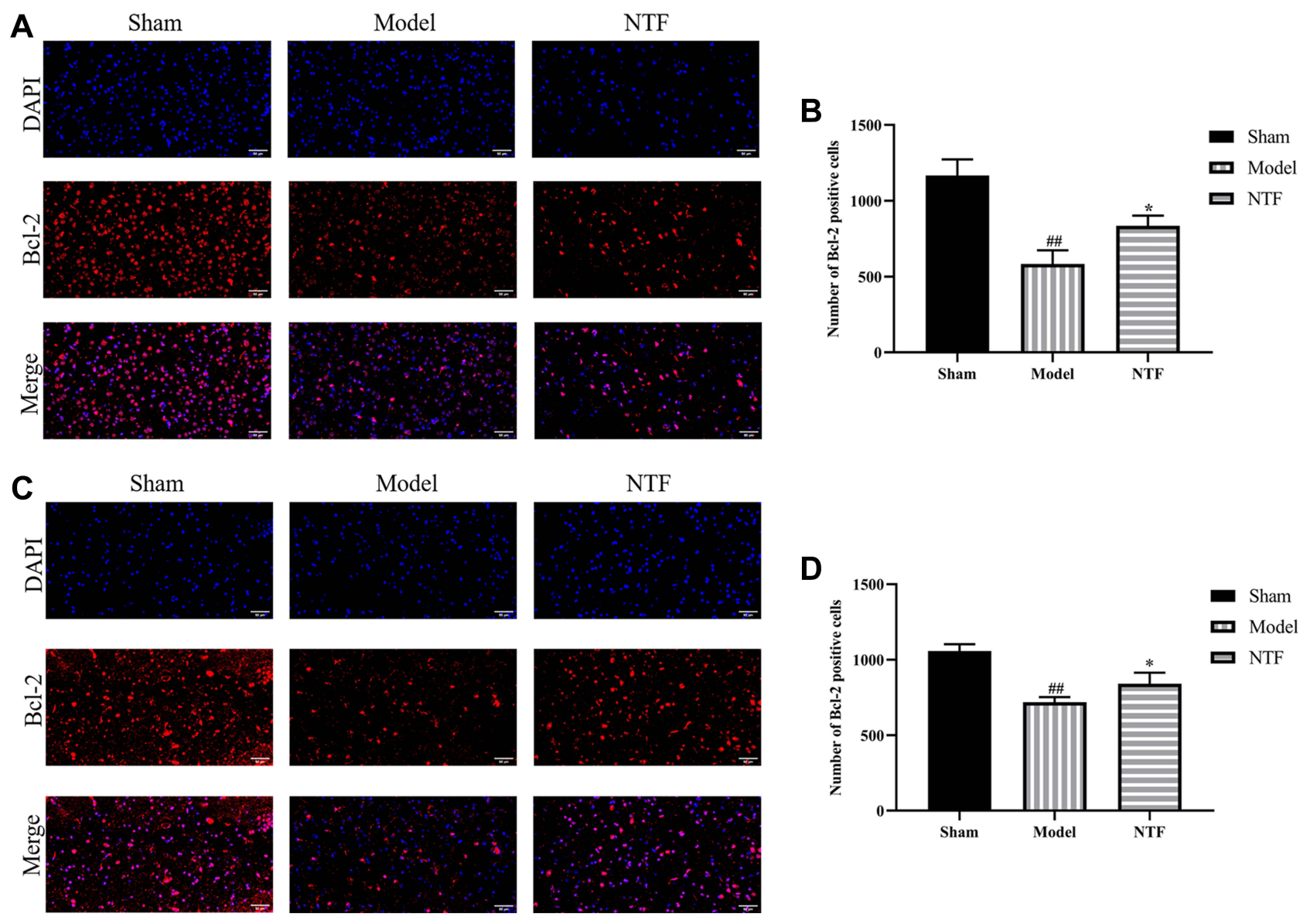

Figure 13 Effects of NTF 7 days prior to CIRI on expression of Bcl-2 of the ischemic cortex and hippocampus in rats $24 \mathrm{~h}$ after reperfusion. (A) Representative images magnified 400 times in ischemic cortex sections examined with specific antibody against Bcl-2 (red); nuclei were stained with DAPI (blue). (B) The numbers of Bcl-2 positive cells in each group after $24 \mathrm{~h}$ of reperfusion in ischemic cortex. (C) Representative images magnified 400 times in ischemic hippocampus sections. (D) The numbers of Bcl-2 positive cells in each group after $24 \mathrm{~h}$ of reperfusion in ischemic hippocampus. All data were presented as mean $\pm \mathrm{SD}$. ${ }^{\# \#} p<0.01$ versus sham group; ${ }^{*} p<0.05$ versus model group, respectively.

signal activation and transcription. ${ }^{69}$ STAT1 has previously been involved in causing cerebral ischemia injury by modulating transcription and phosphorylation of proteins related to apoptosis and cell death. ${ }^{70}$ A previous study discovered that the activation of STAT6 in both microglia and peripheral cells was conductive to STAT6-afforded protection against ischemic brain injury. $^{71}$ It has been reported that STATs, especially STAT6 and possibly STAT1, increase the transcription of $12 / 15-\mathrm{LOX}$ in neuronal cells, which is a key mediator of oxidative stress, leading to neuronal cell death and vascular leakage. ${ }^{72}$ Cerebral ischemia activates several growth factors, cytokines, and their receptor JAK, which recruits STAT3 protein for tyrosine phosphorylation. A dimer consisting of two phosphorylated STAT3 can translocate into the nucleus, consequently regulating the transcription of target genes by binding to DNA. ${ }^{73}$ Meanwhile, The activation of STAT3 has been reportedly associated with the neuroinflammation in cerebral ischemia, ${ }^{74}$ and it could regulate the expression of inflammation-related genes encoding proteins. ${ }^{64}$ STAT3-mediated cytokine signaling regulates gliogenesis and neurogenesis during brain development, thereby exerting neuroprotective effects. $^{75}$ Thus, we here investigated the effects of NTF on the STAT3 signaling pathway. The NTF pretreatment significantly upregulated the expression of p-STAT3 expression in the post-ischemic brains in vivo. However, the role of the STAT3 signaling pathway remains controversial because it has been reported that the expression of p-STAT3 was upregulated in the ischemic penumbra of MCAO mouse brains. ${ }^{76}$ The 

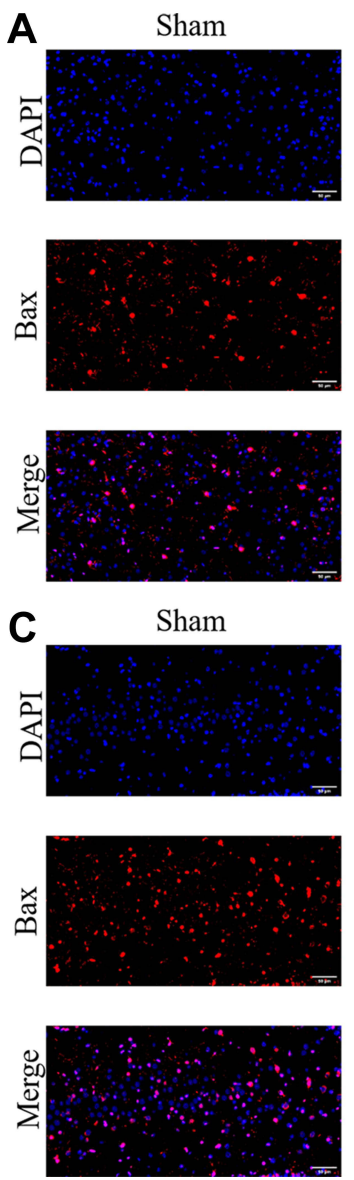

Model
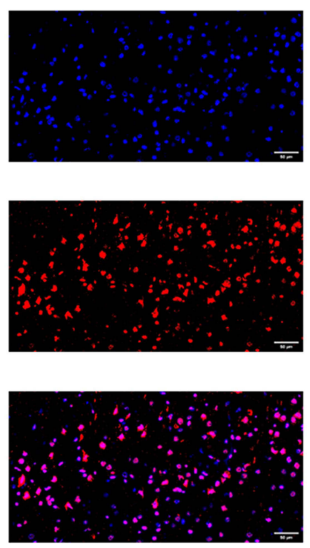

Model
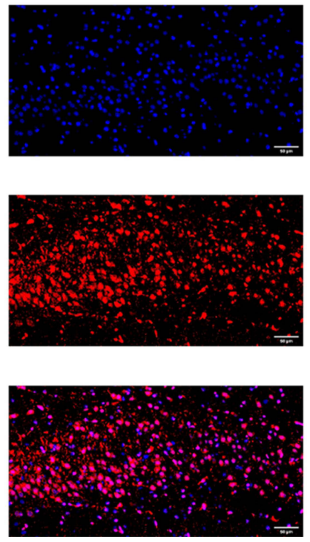
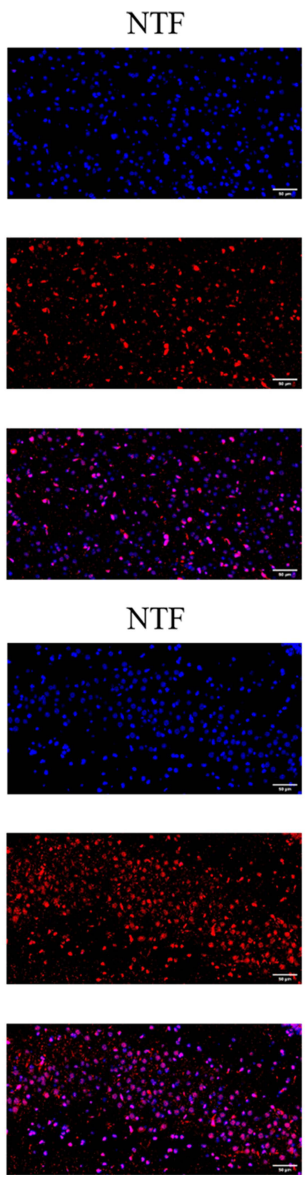

B

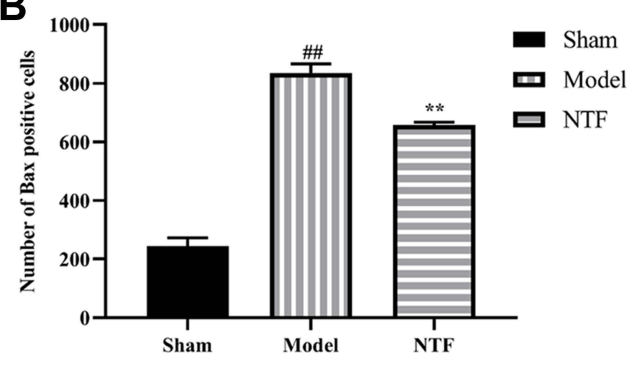

D

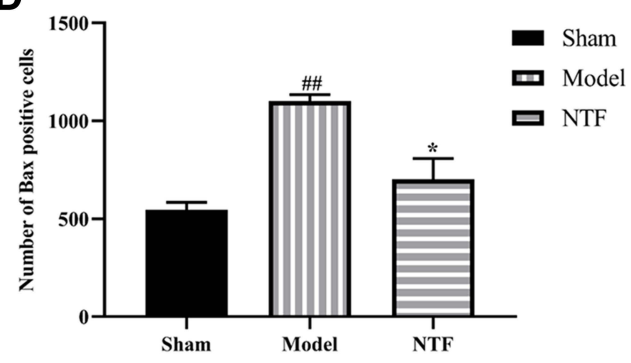

Figure 14 Effects of NTF 7 days prior to CIRI on expression of Bax of the ischemic cortex and hippocampus in rats $24 \mathrm{~h}$ after reperfusion. (A) Representative images magnified 400 times in ischemic cortex sections examined with specific antibody against Bax (red); nuclei were stained with DAPI (blue). (B) The numbers of Bax positive cells in each group after $24 \mathrm{~h}$ of reperfusion in ischemic cortex. (C) Representative images magnified 400 times in ischemic hippocampus sections. (D) The numbers of Bax positive cells in each group after $24 \mathrm{~h}$ of reperfusion in ischemic hippocampus. All data were presented as mean \pm SD. ${ }^{\#} p<0.0$ I versus sham group; ${ }^{* *} p<0.0 \mathrm{I},{ }^{*} p<0.05$ versus model group, respectively.

amphibolous results may result from the difference of species, ischemic duration and location of vascular occlusion. Therefore, the role of STAT1, STAT3 and STAT6 in the pathological mechanisms underlying ischemic stroke and CIRI should be further explored in future studies.

A previous study has shown that inhibitors of PI3K decrease STAT3 phosphorylation, which further suggests that PI3K and STAT3 have some interdependence. ${ }^{77}$ Activated JAKs induce signaling pathways including not only the STAT signaling pathway but also the PI3K/AKT signaling pathway, which plays an important role in mitosis and cell survival. ${ }^{78}$ Previous findings have shown that the PI3K/AKT signaling pathway was involved in cell proliferation, inflammation, differentiation, apoptosis, survival and other activities. ${ }^{79,80}$ The results of our present study showed that the expression of PI3K and p-AKT was reduced in CIRI rats and increased after NTF preadministration, indicating that the PI3K/AKT signaling pathway was activated under NTF pre-treatment conditions. Research illustrated that the survival of neurons after ischemia is related to the activation of the PI3K/ AKT signaling pathway, and the direct consequence is considered to be the suppression of apoptosis and inflammation, ${ }^{81}$ which was basically consistent with our study. Researches have shown that reducing the number of apoptotic cells in ischemic penumbra may be crucial, because the cells here may be salvaged after stroke. ${ }^{82}$ It has also been demonstrated that rehabilitation training can effectively activate the PI3K/ AKT signaling pathway, inhibit neuronal apoptosis, 

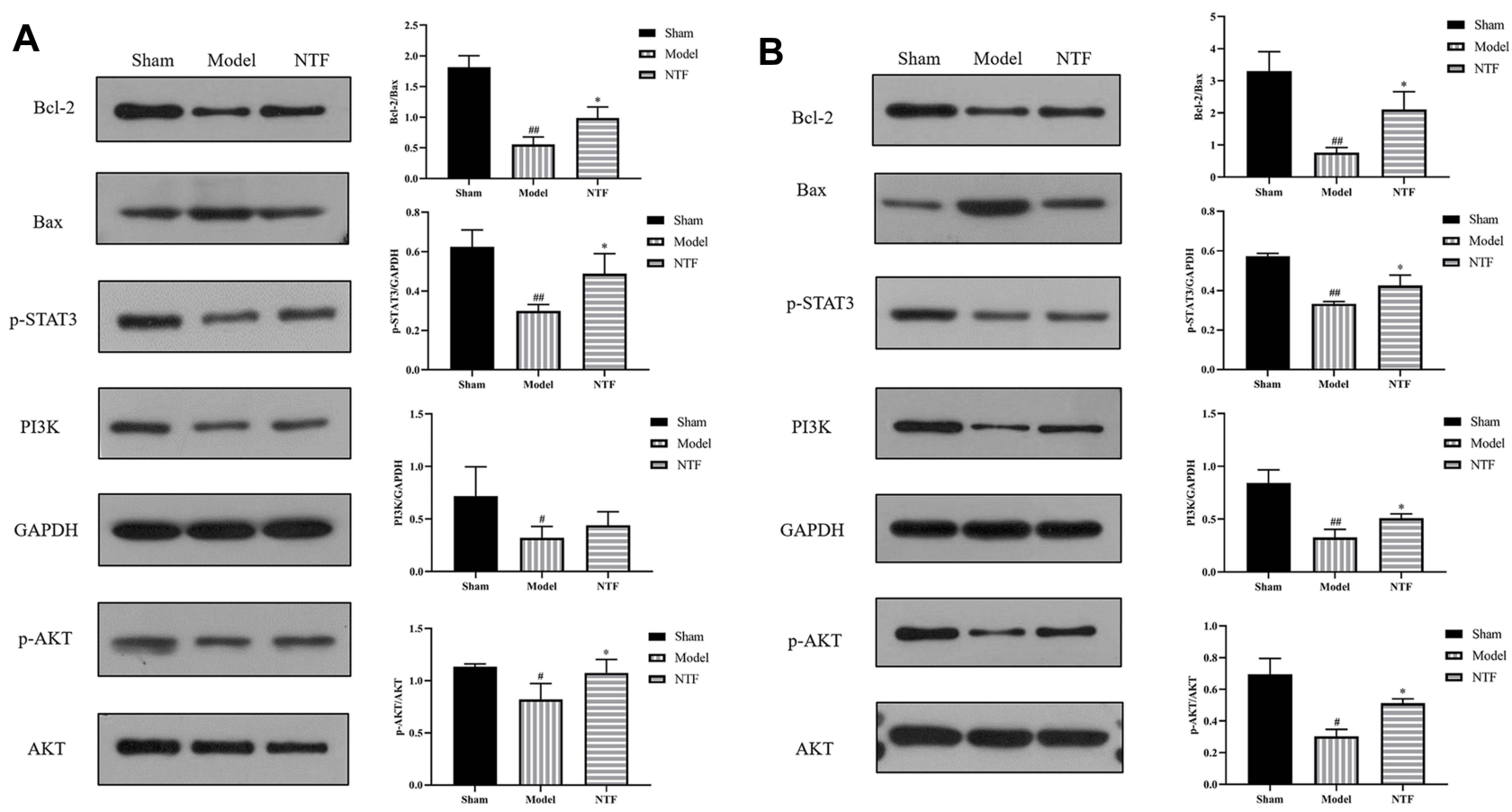

Figure 15 Effects of NTF 7 days prior to CIRI on expression of Bcl-2/Bax, p-STAT3, PI3K and p-AKT/AKT of the ischemic cortex and hippocampus in rats $24 \mathrm{~h}$ after reperfusion by Western blotting. (A) The protein band of Bcl-2/Bax, p-STAT3, PI3K and p-AKT/AKT and corresponding GAPDH in ischemic cortex and corresponding bar graphs. (B) The protein band of Bcl-2/Bax, p-STAT3, PI3K and p-AKT/AKT and corresponding GAPDH in ischemic hippocampus and corresponding bar graphs. All data were presented as mean \pm SD. ${ }^{\# \#} p<0.01,{ }^{\#} p<0.05$ versus sham group; ${ }^{*} p<0.05$ versus model group, respectively.

and promote the recovery of neurological and motor functions in rats with ischemic stroke. ${ }^{83}$ Activated AKT has been found to promote cell survival through phosphorylating. ${ }^{84}$ A previous study showed that activation of the PI3K/AKT signaling pathway mediates the protective effect against neuronal apoptosis after subarachnoid hemorrhage. ${ }^{85} \mathrm{Bcl}-2$, one of the $\mathrm{Bcl}-2$ family proteins, is known as a downstream target of the STAT pathway. ${ }^{86}$ The phosphorylated AKT can activate a variety of apoptosis regulatory proteins, including the pro-apoptotic protein Bax. ${ }^{87}$ Studies indicated that Bcl-2 and Bax, which belong to the Bcl-2 protein family, are crucial proteins in cell apoptosis. Bcl-2 can promote cell survival, while Bax facilitates apoptosis. ${ }^{88}$ In the present study, our data exhibited that NTF decreased the number of apoptotic neuronal cells, raised the expression of $\mathrm{Bcl}-2$ and reduced the expression of Bax, which signified that NTF has an anti-apoptotic effect in CIRI. Taken together, the STAT3/PI3K/AKT signaling pathway may be a critical therapeutic target for reducing apoptosis and enhancing neurogenesis in the treatment of NTF in ischemia stroke and CIRI. Understanding of the biological mechanism of NTF from experimental research may help develop the potential clinical strategy of NTF to treat ischemia stroke and CIRI (Figure 16).

\section{Conclusion}

Taken together, based on the systematic analysis of NTF by the combination of network pharmacology and the experimental verification, our present study showed that pretreatment with NTF protected the brain against ischemic stroke and CIRI via the STAT3/PI3K/AKT signaling pathway. We believe that NTF may serve as a promising therapy strategy for ischemic stroke. In the future, we will conduct further experiments to explore other possible predicted functions and mechanisms, including laser Doppler or laser speckle to observe direct blood flow alterations of cerebral ischemia-reperfusion injury model, through which we will try to provide a theoretical basis for the prevention and treatment of ischemic stroke and CIRI. 


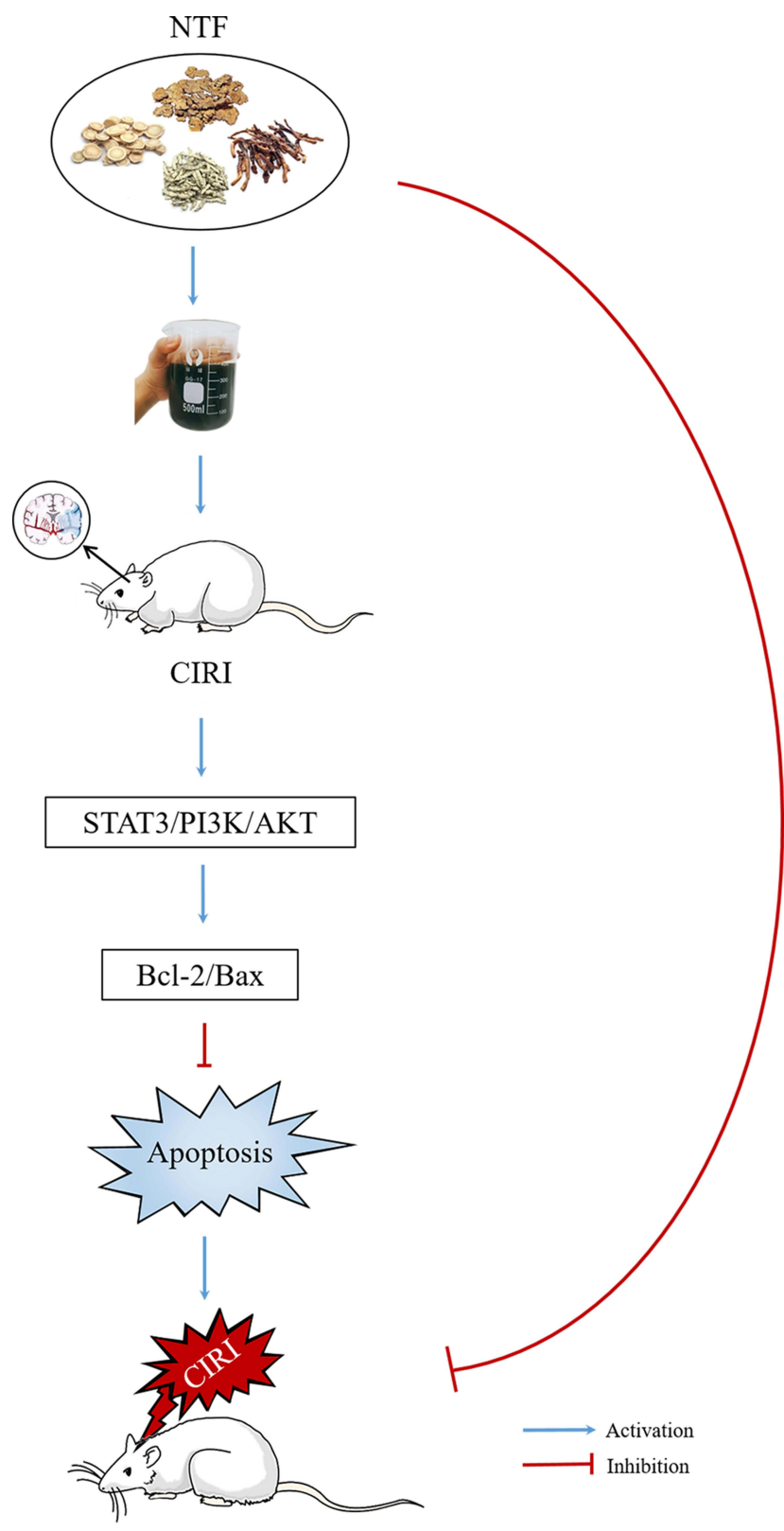

Figure 16 Proposed scheme of NTF suppressed cerebral ischemia-reperfusion induced apoptosis via STAT3/PI3K/AKT signaling pathway. Our results suggest that NTF pretreatment upregulated p-STAT3, PI3K, p-AKT proteins expression, increased the $\mathrm{Bcl}-2 / \mathrm{Bax}$ ratio, alleviated neurological evaluation, reduced infarct volume and apoptosis-positive cells, increased the density of dendritic spines and survival of neurons in cortex and hippocampus sections.

\section{Abbreviations}

CIRI, cerebral ischemia-reperfusion injury; NTF, Naotaifang; OB, oral bioavailability; DL, drug-likeness; GO, gene ontology; KEGG, Kyoto encyclopedia of genes and genomes; rtPA, recombinant tissue plasminogen activator; FDA, American Food and Drug Administration; TCM, traditional Chinese medicine; BYHWD, Buyang Huanwu decoction; HQ, Radix Astragali; CX, Rhizoma Ligustici; DL,
Pheretima; JC, Bombyx Batryticatus; TCMSP, Traditional Chinese Medicine Systems Pharmacology Database and Analysis Platform; OMIM, Online Mendelian Inheritance in Man; DC, degree centrality; $\mathrm{BC}$, betweenness centrality; $\mathrm{CC}$, closeness centrality; HPLC, high performance liquid chromatography; HRMS, high-resolution mass spectrometer; TUNEL, terminal deoxynucleotidyl transferase-mediated dUTP-biotin nick end labeling; SD, standard deviation.

\section{Data Sharing Statement}

The data supporting the findings of this study are available from the corresponding author of this manuscript upon reasonable request and with permission of all the authors.

\section{Ethics Statement}

The animal study was reviewed and approved by the Experimental Animal Health Ethics Committee of Hunan University of Chinese Medicine.

\section{Acknowledgments}

Special thanks to Yanan Luo for English language editing.

\section{Author Contributions}

All authors made substantial contributions to conception and design, acquisition of data, or analysis and interpretation of data; took part in drafting the article or revising it critically for important intellectual content; agreed to submit to the current journal; gave final approval of the version to be published; and agree to be accountable for all aspects of the work.

\section{Funding}

This work was supported by the fund of the National Key Research and Development Program of the Ministry of Science and Technology (2018YFC1704904), and the fund for Youth Top Talent Project of Hubei Provincial Health and Family Planning Commission (EWT-201948), and the Project Supported by Scientific Research Fund of Hunan Provincial Education Department (20A366).

\section{Disclosure}

The authors declare that there are no conflicts of interest for this work.

\section{References}

1. Wu SM, Wu B, Liu M, et al. Stroke in China: advances and challenges in epidemiology, prevention, and management. Lancet Neurol. 2019;18(4):394-405. 
2. Benjamin EJ, Muntner P, Alonso A, et al. Heart disease and stroke statistics-2019 update: a report from the American Heart Association. Circulation. 2019;139(10): e56-e528.

3. Liu S, Levine SR, Winn HR. Targeting ischemic penumbra part I: from pathophysiology to therapeutic strategy. J Exp Stroke Transl Med. 2010;3(1):47-55.

4. Rabinstein AA. Treatment of acute ischemic stroke. Contin Lifelong Learn Neurol. 2017;23(1):62-81.

5. Lahr MMH, Luijckx GJ, Vroomen PCAJ, Van Der Zee DJ, Buskens E. Proportion of patients treated with thrombolysis in a centralized versus a decentralized acute stroke care setting. Stroke. 2012;43(5):1336-1340.

6. Zhou YF, Yan SJ, Song XY, et al. Intravenous thrombolytic therapy for acute ischemic stroke in Hubei, China: a survey of thrombolysis rate and barriers. BMC Neurol. 2019;19(1):202.

7. Mei ZG, Huang YG, Feng ZT, et al. Electroacupuncture ameliorates cerebral ischemia/reperfusion injury by suppressing autophagy via the SIRT1-FOXO1 signaling pathway. Aging (Albany NY). 2020;12 (13):13187-13205.

8. Du LP, Mei ZG, Huang YG, et al. Protection of the Geum japonicum Thunb. var. chinense extracts against oxygen-glucose deprivation and re-oxygenation induced astrocytes injury via BDNF/ PI3K/Akt/CREB pathway. Biomed Pharmacother. 2020;127: $110-123$.

9. Zhang B, Wang X, Li S. An integrative platform of TCM network pharmacology and its application on a herbal formula, Qing-Luo-Yin. Evid Based Compl Altern Med. 2013;2013:456747.

10. Li HY, Zhao LH, Zhang B, et al. A network pharmacology approach to determine active compounds and action mechanisms of Ge-GenQin-Lian decoction for treatment of type 2 diabetes. Evid Based Compl Altern Med. 2014;2014:495840.

11. Zhang YQ, Bai M, Zhang B, et al. Uncovering pharmacological mechanisms of Wu-tou decoction acting on rheumatoid arthritis through systems approaches: drug-target prediction, network analysis and experimental validation. Sci Rep. 2015;30(5):9463.

12. Wang P, Shao BZ, Deng ZQ, et al. Autophagy in ischemic stroke. Prog Neurobiol. 2018;163(1):98-117.

13. Ren $\mathrm{CH}$, Wang B, Li N, Jin KL, Ji XM. Herbal formula Danggui-Shaoyao-San promotes neurogenesis and angiogenesis in rat following middle cerebral artery occlusion. Aging Dis. 2015;6 (4):245-253.

14. Loh KP, Qi J, Tan BKH, Liu XH, Wei BG, Zhu YZ. Leonurine protects middle cerebral artery occluded rats through antioxidant effect and regulation of mitochondrial function. Stroke. 2010;41 (11):2661-2668.

15. Wang HW, Liou KT, Wang YH, et al. Deciphering the neuroprotective mechanisms of Bu-yang Huan-wu decoction by an integrative neurofunctional and genomic approach in ischemic stroke mice. $J$ Ethnopharmacol. 2011;138(1):22-33.

16. Liao J, Xia X, Wang GZ, Shi YM, Ge JW. Naotaifang extract treatment results in increased ferroportin expression in the hippocampus of rats subjected to cerebral ischemia. Mol Med Rep. 2015;11 (6):4047-4052.

17. Hu MB, Yu ZJ, Wang JL, et al. Traditional uses, origins, chemistry and pharmacology of bombyx batryticatus: a review. Molecules. 2017;22(10):1-24.

18. Zhang J, Zou HY, Zhang QX, et al. Effects of Xiaoshuan enteric-coated capsule on neurovascular functions assessed by quantitative multiparametric MRI in a rat model of permanent cerebral ischemia. BMC Complement Altern Med. 2016;16(1):1-14.

19. Lan B, Ge JW, Cheng SW, et al. Extract of Naotaifang, a compound Chinese herbal medicine, protects neuron ferroptosis induced by acute cerebral ischemia in rats. J Integr Med. 2020;18(4):344-350.

20. He YH, Hao XY, Ge JW. Clinical study of naotai formula in treating cerebral infarction with Qi deficiency and blood stasis syndrome. Zhong Guo Zhong Yi Ji Zheng. 2001;10(6):1-4.
21. Zhong Q, Tong J, Zhong XC, Wu DS, Zhu D, Ge JW. Effects of Naotaifang extracts on nerve regeneration after cerebral ischemia reperfusion in rats and influence on Notch 1 protein expression. Zhong Hua Zhong Yi Yao Xue Kan. 2016;34(8):3-6.

22. Chen Y, Zhu HB, Liao J, et al. Regulation effect of Naotai formula on HIF-1 $\alpha /$ VEGF in rats with cerebral ischemia reperfusion. Zhong Guo Zhong Xi Yi Jie He. 2014;34:1225.

23. Huang J, Liao J, Peng XW, et al. Effect of Naotai formula on the expression of Nrf2, HO-1 and membrane iron transport helper protein in hippocampus of rats with cerebral ischemia/reperfusion. Zhong Guo Yao Li Xue Tong Bao. 2017;33(10):1467-1472.

24. Boezio B, Audouze K, Ducrot P, Taboureau O. Network-based approaches in pharmacology. Mol Inform. 2017;36(10):1-10.

25. Szklarczyk D, Morris JH, Cook H, et al. The STRING database in 2017: quality-controlled protein-protein association networks, made broadly accessible. Nucleic Acids Res. 2017;45(1):362-368.

26. Smoot ME, Ono K, Ruscheinski J, Wang PL, Ideker T. Cytoscape 2.8: new features for data integration and network visualization. Bioinformatics. 2011;27(3):431-432.

27. Tang Y, Li M, Wang J, Pan Y, Wu FX. CytoNCA: a cytoscape plugin for centrality analysis and evaluation of protein interaction networks. BioSystems. 2015;127:67-72.

28. Wan YX, Xu L, Liu ZY, et al. Utilising network pharmacology to explore the underlying mechanism of Wumei Pill in treating pancreatic neoplasms. BMC Complement Altern Med. 2019;19(1):1-12.

29. Eswari JS, Dhagat S, Kaser S. Homology modeling and molecular docking studies of Bacillomycin and Iturin synthetases with novel ligands for the production of therapeutic lipopeptides. Curr Drug Discov Technol. 2018;15(2):132-141.

30. Halgren T. New method for fast and accurate binding-site identification and analysis. Chem Biol Drug Des. 2007;69(2):146-148.

31. Halgren TA. Identifying and characterizing binding sites and assessing druggability. J Chem Inf Model. 2009;49(2):377-389.

32. Li XH, Hou Y, Wang XB, et al. To elucidate the inhibition of excessive autophagy of rhodiola crenulata on exhaustive exercise-induced skeletal muscle injury by combined network pharmacology and molecular docking. Biol Pharm Bull. 2020;43(2):296-305.

33. Özçelik AB, Özdemir Z, Sari S, Utku S, Uysal M. A new series of pyridazinone derivatives as cholinesterases inhibitors: synthesis, in vitro activity and molecular modeling studies. Pharmacol Rep. 2019;71(6):1253-1263.

34. Chen L, Zhang YH, Wang S, Zhang Y, Huang T, Cai YD. Prediction and analysis of essential genes using the enrichments of gene ontology and KEGG pathways. PLoS One. 2017;12(9):1-22.

35. Ou BJ, Tao W, Yang SB, et al. The antiapoptosis effect of Geum japonicum thunb. var. chinense extracts on cerebral ischemia reperfusion injury via PI3K/Akt pathway. Evidence-Based Complement Altern Med. 2018;11:1-13.

36. Longa EZ, Weinstein PR, Carlson S, Cummins R. Reversible middle cerebral artery occlusion without craniectomy in rats. Stroke. 1989;20 (1):84-91.

37. Gibb R, Kolb B. A method for vibratome sectioning of Golgi-Cox stained whole rat brain. J Neurosci Methods. 1998;79(1):1-4.

38. Sun MS, Hang J, Sun X, et al. Free radical damage in ischemia-reperfusion injury: an obstacle in acute ischemic stroke after revascularization therapy. Oxid Med Cell Longev. 2018;1(31):1-17.

39. Soares ROS, Losada DM, Jordani MC, Évora P, Castro-E-Silva O. Ischemia/reperfusion injury revisited: an overview of the latest pharmacological strategies. Int J Mol Sci. 2019;20(20):5034.

40. Wu MY, Yiang GT, Liao WT, et al. Current mechanistic concepts in ischemia and reperfusion injury. Cell Physiol Biochem. 2018;46 (4):1650-1667.

41. Ornellas FM, Ornellas DS, Martini SV, et al. Bone marrow-derived mononuclear cell therapy accelerates renal ischemia-reperfusion injury recovery by modulating inflammatory, antioxidant and apoptotic related molecules. Cell Physiol Biochem. 2017;41(5):1736-1752. 
42. Zhang Y, Zhang Y, Jin XF, et al. The role of Astragaloside IV against cerebral ischemia/reperfusion injury: suppression of apoptosis via promotion of P62-LC3-autophagy. Molecules. 2019;24(9):1838.

43. Dai YY, Zhang HJ, Zhang JP, Yan MG. Isoquercetin attenuates oxidative stress and neuronal apoptosis after ischemia/reperfusion injury via Nrf2-mediated inhibition of the NOX4/ROS/NF- $\mathrm{BB}$ pathway. Chem Biol Interact. 2018;284:32-40.

44. Khandelwal P, Yavagal DR, Sacco RL. Acute ischemic stroke intervention. J Am Coll Cardiol. 2016;67(22):2631-2644.

45. Han JY, Li Q, Ma ZZ, Fan JY. Effects and mechanisms of compound Chinese medicine and major ingredients on microcirculatory dysfunction and organ injury induced by ischemia/reperfusion. Pharmacol Ther. 2017;177:146-173.

46. Li LT, Qiu HY, Liu MM, Cai YM. A network pharmacology-based study of the molecular mechanisms of shaoyao-gancao decoction in treating Parkinson's disease. Interdiscip Sci. 2020;12(2):131-144.

47. Yan XL, Yu AM, Zheng HZ, Wang SX, He YY, Wang LS. Calycosin7-O- $\beta$-D-glucoside attenuates OGD/R-induced damage by preventing oxidative stress and neuronal apoptosis via the SIRT1/FOXO1/PGC$1 \alpha$ pathway in HT22 cells. Neural Plast. 2019;2019:8798069.

48. Wang HL, Zhou QH, Xu MB, Zhou XL, Zheng GQ. Astragaloside IV for experimental focal cerebral ischemia: preclinical evidence and possible mechanisms. Oxid Med Cell Longev. 2017;2017:8424326.

49. Koh PO. Ferulic acid attenuates focal cerebral ischemia-induced decreases in p70S6 kinase and S6 phosphorylation. Neurosci Lett. 2013;555:7-11.

50. Ding Y, Du J, Cui F, Chen L, Li K. The protective effect of ligustrazine on rats with cerebral ischemia-reperfusion injury via activating PI3K/Akt pathway. Hum Exp Toxicol. 2019;38(10):1168-1177.

51. Huang CQ, Li W, Zhang QF, et al. Anti-inflammatory activities of Guang-Pheretima extract in lipopolysaccharide-stimulated RAW 264.7 murine macrophages. BMC Complement Altern Med. 2018;18 (1):1-11.

52. Wu YL, Hu SN, Ma YN, et al. Novel Pheretima guillelmi-derived antithrombotic protein DPf3: identification, characterization, in vitro evaluation and antithrombotic mechanisms investigation. Int $\mathrm{J} \mathrm{Biol}$ Macromol. 2020;154:545-556.

53. Kwon HC, Lee KC, Cho OR, et al. Sphingolipids from Bombycis Corpus 101A and their neurotrophic effects. J Nat Prod. 2003;66 (4):466-469.

54. Blake JA, Christie KR, Dolan ME, et al. Gene ontology consortium: going forward. Nucleic Acids Res. 2015;43(1):1049-1056.

55. Kanehisa M, Sato Y, Kawashima M, Furumichi M, Tanabe M. KEGG as a reference resource for gene and protein annotation. Nucleic Acids Res. 2016;44(1):457-462.

56. Sheng LL, Mao XY, Yu QX, Yu D. Effect of the PI3K/AKT signaling pathway on hypoxia-induced proliferation and differentiation of bone marrow-derived mesenchymal stem cells. Exp Ther Med. 2017;13 (1):55-62.

57. Pan BS, Wang YK, Lai MS, Mu YF, Huang BM. Cordycepin induced MA-10 mouse Leydig tumor cell apoptosis by regulating p38 MAPKs and PI3K/AKT signaling pathways. Sci Rep. 2015;5:13372.

58. Tian YS, Zhong D, Liu QQ, et al. Upregulation of miR-216a exerts neuroprotective effects against ischemic injury through negatively regulating JAK2/STAT3-involved apoptosis and inflammatory pathways. J Neurosurg. 2018;130(3):977-988.

59. Hou YY, Wang K, Wan WJ, Cheng Y, Pu X, Ye XF. Resveratrol provides neuroprotection by regulating the JAK2/STAT3/PI3K/ AKT/mTOR pathway after stroke in rats. Genes Dis. 2018;5 (3):245-255.

60. Jiao SJ, Zhu HC, He P, Teng JF. Betulinic acid protects against cerebral ischemia/reperfusion injury by activating the PI3K/Akt signaling pathway. Biomed Pharmacother. 2016;84:1533-1537.

61. Zhang HF, Xiong XX, Liu J, et al. Emulsified isoflurane protects against transient focal cerebral ischemia injury in rats via the PI3K/ Akt signaling pathway. Anesth Analg. 2016;122(5):1377-1384.
62. Zhang YH, Liu JX, Yang B, et al. Ginkgo biloba extract inhibits astrocytic lipocalin-2 expression and alleviates neuroinflammatory injury via the JAK2/STAT3 pathway after ischemic brain stroke. Front Pharmacol. 2018;9:518.

63. Palareti G, Legnani C, Cosmi B, et al. Comparison between different D-Dimer cutoff values to assess the individual risk of recurrent venous thromboembolism: analysis of results obtained in the DULCIS study. Int J Lab Hematol. 2016;38(1):42-49.

64. Li L, Sun LL, Qiu Y, Zhu WJ, Hu KY, Mao JQ. Protective effect of stachydrine against cerebral ischemia-reperfusion injury by reducing inflammation and apoptosis through P65 and JAK2/STAT3 signaling pathway. Front Pharmacol. 2020;11:1-14.

65. Todd L, Squires N, Suarez L, Fischer AJ. Jak/Stat signaling regulates the proliferation and neurogenic potential of Müller glia-derived progenitor cells in the avian retina. Sci Rep. 2016;6:35703.

66. Müller S, Chakrapani BPS, Schwegler H, Hofmann HD, Kirsch M. Neurogenesis in the dentate gyrus depends on ciliary neurotrophic factor and signal transducer and activator of transcription 3 signaling. Stem Cells. 2009;27(2):431-441.

67. Hua Y, Pardoll D, Jove R. STATs in cancer inflammation and immunity: a leading role for STAT3. Nat Rev Cancer. 2009;9(11):798-809.

68. Xin $\mathrm{P}, \mathrm{Xu} \mathrm{XY}$, Deng $\mathrm{CJ}$, et al. The role of JAK/STAT signaling pathway and its inhibitors in diseases. Int Immunopharmacol. 2020;80:106210.

69. Morris R, Kershaw NJ, Babon JJ. The molecular details of cytokine signaling via the JAK/STAT pathway. Protein Sci. 2018;27 (12):1984-2009.

70. Takagi Y, Harada J, Chiarugi A, Moskowitz MA. STAT1 is activated in neurons after ischemia and contributes to ischemic brain injury. J Cereb Blood Flow Metab. 2002;22(11):1311-1318.

71. Cai W, Dai XJ, Chen J, et al. STAT6/Arg1 promotes microglia/ macrophage efferocytosis and inflammation resolution in stroke mice. JCI Insight. 2019;4(20):1-20.

72. Jung JE, Karatas H, Liu Y, et al. STAT-dependent upregulation of 12/ 15-lipoxygenase contributes to neuronal injury after stroke. J Cereb Blood Flow Metab. 2015;35(12):2043-2051.

73. Snyder M, Huang XY, Zhang JJ. Stat3 is essential for neuronal differentiation through direct transcriptional regulation of the Sox6 gene. FEBS Lett. 2011;585(1):148-152.

74. McGuckin CP, Jurga M, Miller AM, et al. Ischemic brain injury: a consortium analysis of key factors involved in mesenchymal stem cell-mediated inflammatory reduction. Arch Biochem Biophys. 2013;534(1-2):88-97.

75. White CW, Fan XL, Maynard JC, et al. Age-related loss of neural stem cell O-GlcNAc promotes a glial fate switch through STAT3 activation. Proc Natl Acad Sci U S A. 2020;117(36):22214-22224.

76. Zhu H, Jian ZH, Zhong Y, et al. Janus kinase inhibition ameliorates ischemic stroke injury and neuroinflammation through reducing NLRP3 inflammasome activation via JAK2/STAT3 pathway inhibition. Front Immunol. 2021;22(12):1-16.

77. Lin CC, Chen SY, Lien HY, Lin SZ, Lee TM. Targeting the PI3K/ STAT3 axis modulates age-related differences in macrophage phenotype in rats with myocardial infarction. J Cell Mol Med. 2019;23 (9):6378-6392.

78. Stark GR, Cheon H, Wang Y. Responses to cytokines and interferons that depend upon JAKs and STATs. Cold Spring Harb Perspect Biol. 2018;10(1):1-16.

79. Zhao SF, Fu JD, Liu XR, Wang T, Zhang JL, Zhao YL. Activation of Akt/GSK-3beta/beta-catenin signaling pathway is involved in survival of neurons after traumatic brain injury in rats. Neurol Res. 2012;34(4):400-407.

80. Lim W, Yang C, Bazer FW, Song G. Luteolin inhibits proliferation and induces apoptosis of human placental choriocarcinoma cells by blocking the PI3K/AKT pathway and regulating sterol regulatory element binding protein activity. Biol Reprod. 2016;95 (4):82. 
81. Zhou ZH, Xu NB, Matei N, et al. Sodium butyrate attenuated neuronal apoptosis via GPR41/G $\beta \gamma / \mathrm{PI} 3 \mathrm{~K} / \mathrm{Akt}$ pathway after MCAO in rats. J Cereb Blood Flow Metab. 2021;41(2):267-281.

82. Radak D, Katsiki N, Resanovic I, et al. Apoptosis and acute brain ischemia in ischemic stroke. Curr Vasc Pharmacol. 2017;15(2):115-122.

83. Jin XF, Wang S, Shen M, et al. Effects of rehabilitation training on apoptosis of nerve cells and the recovery of neural and motor functions in rats with ischemic stroke through the PI3K/Akt and Nrf2/ ARE signaling pathways. Brain Res Bull. 2017;134:236-245.

84. Ebner M, Lučić I, Leonard TA, Yudushkin I. PI(3,4,5)P3 engagement restricts Akt activity to cellular membranes. Mol Cell. 2017;65 (3):416-431.
85. Zhuang Z, Zhao XD, Wu Yet al. The anti-apoptotic effect of PI3K-akt signaling pathway after subarachnoid hemorrhage in rats. Ann Clin Lab Sci. 2011;41(4):364-372.

86. Tang H, Gamdzyk M, Huang L, et al. Delayed recanalization after MCAO ameliorates ischemic stroke by inhibiting apoptosis via HGF/ c-Met/STAT3/Bcl-2 pathway in rats. Exp Neurol. 2020;330:113359.

87. Chen BS, Shen ZJ, Wu DG, et al. Glutathione peroxidase 1 promotes NSCLC resistance to cisplatin via ROS-induced activation of PI3K/ AKT pathway. Biomed Res Int. 2019;2019:7640547.

88. Kim KB, Lee S, Kim JH. Neuroprotective effects of urolithin a on $\mathrm{H}_{2} \mathrm{O}_{2}$-induced oxidative stress-mediated apoptosis in SK-N-MC cells. Nutr Res Pract. 2020;14(1):3-11.

\section{Publish your work in this journal}

Drug Design, Development and Therapy is an international, peerreviewed open-access journal that spans the spectrum of drug design and development through to clinical applications. Clinical outcomes, patient safety, and programs for the development and effective, safe, and sustained use of medicines are a feature of the journal, which has also been accepted for indexing on PubMed Central. The manuscript management system is completely online and includes a very quick and fair peer-review system, which is all easy to use. Visit http://www. dovepress.com/testimonials.php to read real quotes from published authors. 